\title{
The Role of Probe-Trial Distractors in the Production/Removal of the Spatial Negative Priming Effect
}

Peter D. Haworth, The University of Western Ontario

Supervisor: Dr. Eric Buckolz, The University of Western Ontario

A thesis submitted in partial fulfillment of the requirements for the Master of Arts degree in

Kinesiology

(c) Peter D. Haworth 2013

Follow this and additional works at: https://ir.lib.uwo.ca/etd

Part of the Cognition and Perception Commons, and the Sports Studies Commons

\section{Recommended Citation}

Haworth, Peter D., "The Role of Probe-Trial Distractors in the Production/Removal of the Spatial Negative Priming Effect" (2013). Electronic Thesis and Dissertation Repository. 1436.

https://ir.lib.uwo.ca/etd/1436

This Dissertation/Thesis is brought to you for free and open access by Scholarship@Western. It has been accepted for inclusion in Electronic Thesis and Dissertation Repository by an authorized administrator of Scholarship@Western. For more information, please contact wlswadmin@uwo.ca. 
THE ROLE OF PROBE-TRIAL DISTRACTORS IN THE PRODUCTION/REMOVAL OF THE SPATIAL NEGATIVE PRIMING EFFECT

(Thesis format: Integrated Article)

by

Peter David $\underline{\text { Haworth }}$

Graduate Program in Kinesiology

A thesis submitted in partial fulfillment of the requirements for the degree of Master of Arts

The School of Graduate and Postdoctoral Studies

The University of Western Ontario

London, Ontario, Canada

(C) Peter David Haworth 2013 


\section{Abstract}

In spatial negative priming (SNP) tasks, trials are presented in pairs; first the 'prime', and then the 'probe'. Target and/or distractor events appear on both trials and probe target reaction time is significantly lengthened when it arises at a former distractor-occupied location (ignored-repetition [IR] trial), relative to when it appears at a new location (control [CO] trial). This latency inequality, which is not inevitable, defines the SNP effect. Here, we examined the influence of prime and probe trial distractor identity similarity on restoring the SNP effect when its prevention was successfully motivated by the use of a .25 (distractor present)/.75 (distractor absent) condition. Two results were important: (1) the SNP effect was restored when the probe distractor identity fully matched that of the prime trial, but not when distractor identities partially or totally mismatched, showing a retrieval role for the probe distractor, and, (2) target-repeat trial facilitation showed the same pattern, present with full matches, otherwise being absent. These results showed that prime-trial processing representations are stored episodically in location tasks, and that event identities are part of the episode, making distractor event identity matches critical for prime representation retrieval. Additionally, event numbers were not part of the episode so that matching event numbers between prime and probe trials was not important for retrieval of stored prime representations.

Keywords: Spatial Negative Priming, Modulation, Distractor Processing, Inhibitory Aftereffects 


\section{Co-authorship}

This thesis contains material that was submitted for publication. The principle author of this manuscript was Peter Haworth, co-authored by Dr. Eric Buckolz and Ben Kajaste 


\section{Acknowledgments}

I would like to thank my parents, Robert and Julie, for their continued support with my postgraduate studies. I understand it must have been difficult allowing your child to study on a different continent but I've been looked after very well by our colonial cousins. I'd like to express my gratitude to my fiancée, Chloé, for her tremendous support both emotionally and financially through my Canadian endeavour. Chloé's support has been invaluable and I'm sure to repay the favour someday.

Dr. Buckolz, you've been a fantastic supervisor and I appreciate everything you've done for me. You're one of few, which can express theoretical issues and still persist with an ongoing 'gag'. It's been a pleasure working with you and I'm sure we'll keep in contact over the coming years.

I'd also like to thank Ben Kajaste and Alexandra Stoddart for their entertainment and support during the day-to-day grind. Additionally, thanks must go to Jenn Plaskett, Jacqui Saunders and the Faculty of Health Sciences for all their support during my time at Western University.

Finally, I would like to thank my committee members, Dr. Craig Hall and Dr. Don Morrow, as well as my examination board members, Dr. Craig Hall, Dr. Jeff Holmes and Dr. Alan Salmoni. 


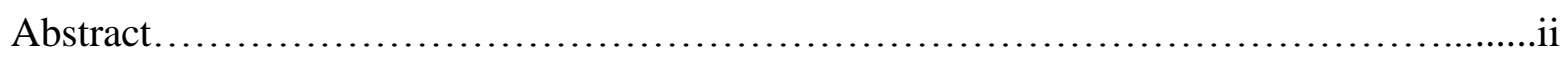

Co-Authorship Statement..............................................................

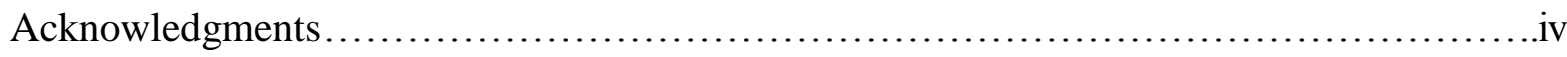

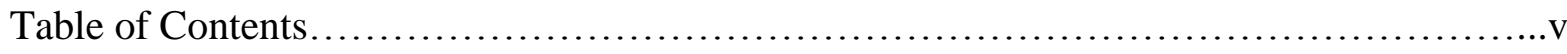

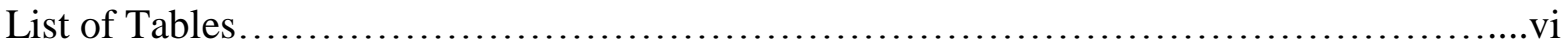

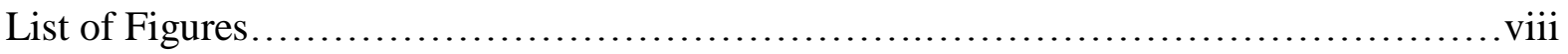

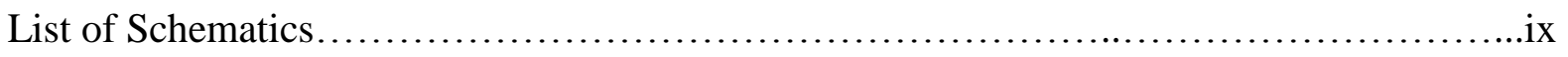

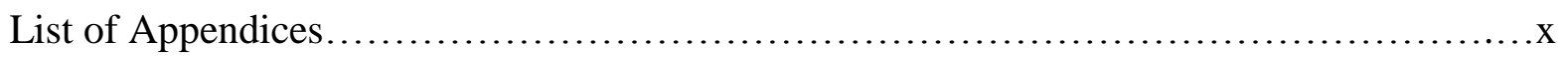

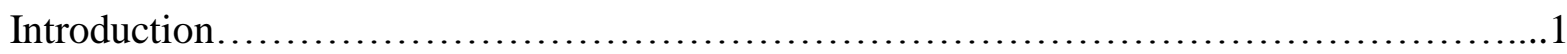

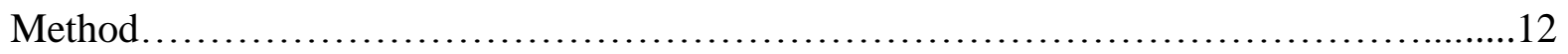

Participants....................................................... 12

Apparatus............................................................ 12

Procedure.............................................................

Design.............................................................

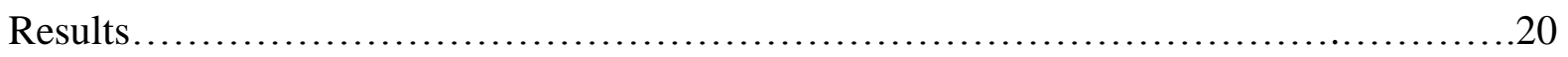

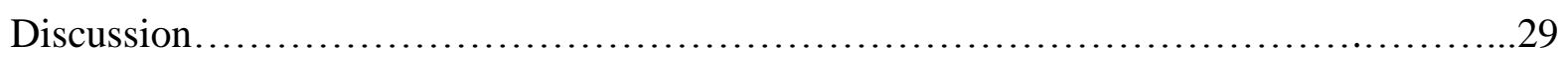

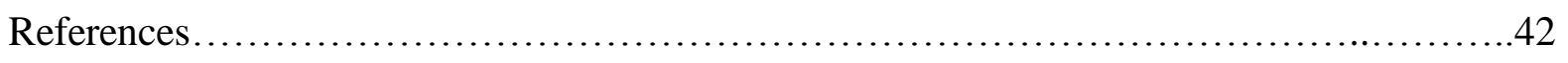

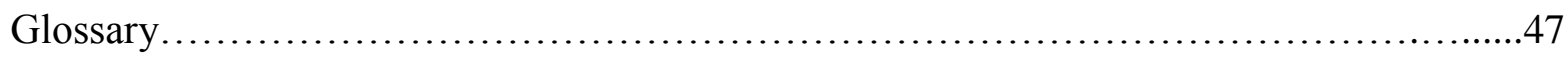

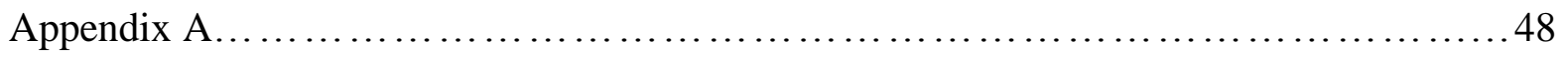

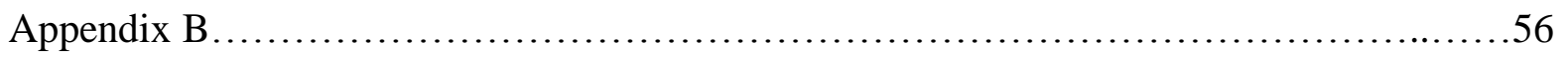

Appendix C

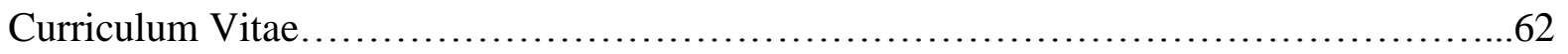




\section{List of Tables}

Table Description

Page

Prime-probe trial target/distractor combinations used in prior spatial negative priming tasks, along with various probabilities related to probe distractor appearance. Note: $\mathrm{T}+\mathrm{D}=$ target plus distractor; $\mathrm{T}$-only $=$ targets only. $1.00=$ certain probe trial distractor, $.75 / .25=$ probe trial distractor present/distractor absent, $.50 / .50=$ probe trial equi-probable distractor present/distractor absent, $.25 / .75=$ probe trial distractor present/distractor absent, $1.00=$ certain probe trial distractor absent. $* .20 / .80$ ratio. $* *=$ utilized peripheral event locations...........................................................2

2 Distractor identity matching levels (full match, partial match, total mismatch: between-subjects), each produced in two ways by using different prime and probe trial distractor identities (between-subjects: Subgroups). ${ }^{\text {a }} 20$ participants within each condition but only experienced one type of prime-probe distractor identity matching level. ${ }^{\mathrm{b}} 10$ subjects per distractor identity combination

Mean reaction times (ms) for t-only probes (100\%); pre-and-post experimental task. Note. Spatial Negative Priming = Ignored-repetition Control; Target-repeat Effect $=$ Target-repeat - Control. ()$=$ standard error $(\mathrm{ms}) ;[]=$ button press error percentage. ${ }^{*} \mathrm{p}<0.05 . \mathrm{n}$

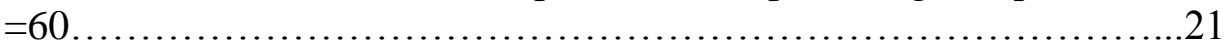

Mean reaction times (RT, ms) for the Trial Type (ignored-repetition, control, target-repeat) and Distractor Identity Matching levels (full match, partial match, total mismatch) factors Note. Spatial Negative Priming = Ignored-repetition - Control; Target-repeat Effect $=$ Targetrepeat - Control. ( ) = standard error $(\mathrm{ms})$; [ ] = button press error

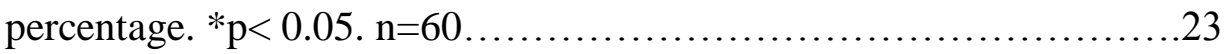

Mean reaction times (RT, ms) for the Trial Type (ignored-repetition, control, target-repeat) and Distractor Identity Matching levels (full match, partial match, total mismatch) factors. Note. Spatial Negative Priming $=$ Ignored-repetition - Control; Target-repeat Effect $=$ Targetrepeat - Control. $(\mathrm{)}=$ standard error $(\mathrm{ms})$; [ ] = button press error percentage. ${ }^{*} \mathrm{p}<0.05 . \mathrm{n}=30$..... .25

Distractor identity matching levels (full match, partial match, total mismatch: within-subjects), each produced in two different conditions by using different prime and probe trial distractor identities (within- 
subjects).30 participants completed both conditions as assigned to their respective groups. All subjects experienced three types of prime-probe distractor identity matching levels within one condition...............51

Mean reaction times (RT, ms) for the Trial Type (ignored-repetition, control, target-repeat) and Distractor Identity Matching levels (full match, partial match, total mismatch) factors Note. Spatial Negative Priming $=$ Ignored-repetition - Control. $(\mathrm{)}=$ standard error $(\mathrm{ms}) ;[\mathrm{]}=$ button press error percentage. ${ }^{*} \mathrm{p}<0.05 . \mathrm{n}=30$ per group between conditions.... .55 


\section{List of Figures}

Figure Description

Page

Mean Probe-trial reaction times as a function of Distractor Identity Similarity levels (full match, partial match, total mismatch), Probe-type (ignored repetition [IR] vs. control [CO]) for target-plus-distractor probe

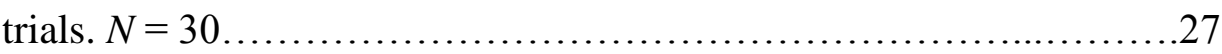

2

Mean Probe-trial reaction times as a function of Distractor Identity Similarity levels (full match, partial match, total mismatch), Probe-type (ignored repetition [IR] vs. control [CO]) for target-only probe trials. $N=$ 30 . .28 
An illustration of the response-based [1] and the object-based [2] pathways via which stored prime-trial distractor processing representations might be retrieved at the time of probe trial event delivery (Buckolz, Edgar, Kajaste, Lok, \& Khan, 2012). Note. $\mathrm{t}=$ target, $\mathrm{d}=$ distractor, $\mathrm{A}=$ activation, $\mathrm{I}=$ inhibition, $\mathrm{ER}=$ execution resistance, IAEs $=$ inhibitory after-effects. The prime-probe trial type shown above elicits an ignored repetition trial.

Screen display of bar markers, fixation cross and stick figure seen by subjects; the letters of the keyboard buttons assigned to each locations are added (DVML) as is "Fixation cross" .13

An illustrative sequence of events for a four-response four location task with a target (white rectangle) plus distractor (black rectangle) prime trial (Panel 3) followed by either a target plus distractor $(T+D)$ or a targetonly probe trial (T-Only) (Panels 6) 


\section{List of Appendices}

Page

Appendix A

Pilot study 46

Purpose... 46

Method 46

Results... 49

Implications

50

Appendix $B$

The University of Western Ontario Research Ethics Board of Approval Notice.

Appendix $C$

Letter of Information... 56

Script. 58 


\section{Introduction}

With the typical spatial negative priming (SNP) procedure of interest here, target and/or distractor events are presented centrally on sequentially related trial pairs; first the 'prime', and then the 'probe'. Most often, probe trial target reaction time is lengthened when it arises at a former distractor-occupied location (i.e., ignored-repetition [IR] trial) relative to when it appears at a previously empty location (i.e., control trial). This latency inequality (RT[IR] > RT[control]) is used most often to identify the presence of the SNP effect (e. g., Buckolz, Boulougouris, \& Khan, 2002; Neill, Terry, \& Valdes, 1994; Tipper, Weaver, Cameron, Brehaut, \& Bastedo, 1991; Guy, Buckolz, \& Pratt, 2004). An ongoing question that we continue to investigate here deals with how the presence or absence of a probe trial distractor influences the presence or absence of the SNP phenomenon (e.g., Chao, 2009).

Prior work on this question has varied the probability of a probe distractor along a continuum ranging from its certain absence (100\%; target-only probe trial) to its certain presence (target + distractor probe trial) [e.g., Buckolz et al., 2002; Fitzgeorge \& Buckolz, 2008; Guy et al., 2004; Guy, Buckolz, \& Fitzgeorge, 2007; Milliken, Tipper, Houghton, \& Lupianez, 2000; see Table 1 for prime-probe probability findings]. As it turns out, the impact that manipulating probe distractor probability has on SNP depends on the event (target/distractor) contents of the preceding prime trial (Guy \& Buckolz, 2007; Guy et al., 2007), and so a summary of this research needs to be set out separately for target + distractor versus distractor-only prime. 


\section{Table 1}

Prime-probe trial target/distractor combinations used in prior spatial negative priming tasks, along with various probabilities related to probe distractor appearance.

\begin{tabular}{|c|c|c|c|c|c|c|c|c|c|c|c|c|c|}
\hline \multirow[b]{3}{*}{$\begin{array}{l}\text { Probe } \\
\text { Configuration }\end{array}$} & \multicolumn{13}{|c|}{ Prime-trial type } \\
\hline & \multirow{2}{*}{$\frac{1.00}{T+D}$} & $.75 / .25$ & $.50 / .50$ & $.25 / .75$ & 1.00 & 1.00 & $.75 / .25$ & & 1.50 & $.25 / .75$ & 1.00 & 1.00 & 1.00 \\
\hline & & $\overline{T+D \quad T \text {-only }}$ & $\overline{T+D \quad T \text {-only }}$ & $\overline{T+D \quad T \text {-only }}$ & $\overline{T-o n l y}$ & $\overline{T+D}$ & $\overline{T+D \quad T \text {-only }}$ & $T+D$ & T-only & $\overline{T+D \quad T-o n l y}$ & $\overline{T-o n l y}$ & $\overline{T+D}$ & T-only \\
\hline $\begin{array}{l}\text { Reference } \\
\text { Number: }\end{array}$ & \multicolumn{5}{|c|}{$\begin{array}{l}\text { 1) Buckolz, Avamidis, \& Fitzgeorge (2008) } \\
\text { 2) Buckolz, Boulougoris, \& Khan (2002) } \\
\text { 3) Buckolz, Edgar, Kajaste, Lok, \& Khan (2012) } \\
\text { 4) Buckolz, Fitzgeorge, \& Knowles (2012) } \\
\text { 5) Buckolz, Goldfarb, \& Khan (2004) } \\
\text { 6) Chao (2009)** } \\
\text { 7) Chao (2011)** } \\
\text { 8) Christie \& Klein (2001)** }\end{array}$} & \multicolumn{6}{|c|}{$\begin{array}{l}\text { 9) Fitzgeorge \& Buckolz (2008 } \\
\text { 10) Guy \& Buckolz (2007) } \\
\text { 11) Guy, Buckolz, \& Fitzgeorge (2007) } \\
\text { 12) Guy, Buckolz, \& Pratt (2004) } \\
\text { 13) Houghton \& Mari-Beffa (2005) } \\
\text { 14) Milliken, Tipper, \& Weaver (1994) } \\
\text { 15) Milliken, Tipper, Houghton, \& Lupianez (2000) } \\
\text { 16) Neill, Terry, \& Valdes (1994) }\end{array}$} & & \\
\hline
\end{tabular}

Note: $\mathrm{T}+\mathrm{D}=$ target plus distractor; $\mathrm{T}$-only $=$ targets only. $1.00=$ certain probe trial distractor, $.75 / .25=$ probe trial distractor present/distractor absent, $.50 / .50$ $=$ probe trial equi-probable distractor present/distractor absent, $.25 / .75=$ probe trial distractor present/distractor absent, $1.00=$ certain probe trial distractor absent. *.20/.80 ratio. $* *$ utilized peripheral event locations. 
Target + Distractor Prime Trials

When the prime trial contains both a target and a distractor, an SNP effect is observed whenever a probe distractor is actually present, irrespective of it's probability of appearing (i.e., $100 \%-25 \%$ ). Alternately, when the probe trial is actually distractor free, the impact on SNP is a function of its probability of appearing. When the probe distractor's absence is certain (100\% target-only probe trial) or highly likely (75\%), an SNP effect is not observed; otherwise, this phenomenon is present (e.g., Buckolz et al., 2002; Buckolz, Avramidis, \& Fitzgeorge, 2008; Fitzgeorge \& Buckolz, 2008; Guy et al., 2004; Neill et al., 1994).

\section{Distractor-only Prime Trials}

When the prime trial predictably or randomly (target or distractor prime design; e.g., Buckolz et al., 2008) contains only a distractor event, the SNP phenomenon is invariably observed; irrespective of probe distractor probability and independent of whether a distractor actually appears or not (e. g., Buckolz et al.; Guy et al., 2004; Milliken et al., 2000). The best illustration of this pattern was presented in a single study by Fitzgeorge and Buckolz (2008). They showed that the experimental manipulations that caused the elimination of the SNP effect (including both probe-trial distractor and ignored-repetition trial probability variations) when the prime trial contained both a target + a distractor, failed to do so when the prime held only a distractor. Relatedly, Guy et al. (2007) showed that the prime target had to cause the actual execution of one of the experimental responses for SNP prevention to occur. Simply preparing to produce an experimental response, or executing a response outside of the experimental subset (i.e., a self-initiated keyboard bar press), did not result in SNP removal.

The next step here is to outline our preferred explanation of the processes that give rise to SNP production, which closely follows versions presented elsewhere (Buckolz, Edgar, Kajaste, Lok, \& Khan, 2012b; Fitzgeorge, Buckolz, \& Khan, 2011), and which takes into 
account the findings of probe distractor manipulations highlighted above. Before doing so, there is an important condition included in the distractor-only prime trial designs that actually sets aside a common and surprisingly persistent misconception that negative priming is necessarily the outcome of selective attention, necessitated when a prime distractor has to be distinguished from an accompanying target stimulus (e.g., Connelly \& Hasher, 1993 ).

Contrary to this view, Milliken et al. (2000), followed by others (Buckolz, et al., 2012b; Fitzgeorge et al., 2011; Guy et al., 2004), showed that distractor-only prime trials followed by target-only probe trials produced an SNP effect. Clearly, the generation of SNP does not require either a prime trial or a probe trial selection. In fact, the size of the SNP effect produced by distractor-only and distractor + target prime trials is the same (Buckolz et al., 2008), indicating that the SNP producing aspect of distractor processing is unaltered, whether a selection has been engaged in or not. Hence, the SNP model outlined below has discarded any reference to selective attention, and this includes the role played by the probe distractor in SNP generation.

\section{A Theoretical Account of Spatial Negative Priming (SNP) Production}

The SNP model set out here is an inhibition-based one, following the broad strokes of the views described by Houghton and Tipper (1994) and Tipper (2001). An inhibition-based approach is recommended because it can explain the majority of existing SNP data as well as or better than competing explanations. Furthermore, some competing negative priming (NP) theories, such as the match/mismatch idea (Park \& Kanwisher, 1994) or the Episodic Retrieval notion (Neill, 1977), have been shown to be lacking as SNP accounts, especially in the case of the former (Milliken et al., 2000; Guy et al., 2004; Tipper, 2001). Additionally, in support of an inhibition-based explanation for the SNP effect, fMRI measurements show 
selective activation of the neural structures responsible for inhibitory processing on ignoredrepetition trials (Bauer, Gebhardt, Ruprecht, Gallhofer, \& Sammer, 2012).

The model begins with the stipulation that distractor-occupied prime trial locations are processed automatically. This is indicated not only because distractor processing occurs in spite of instructions/intentions to the contrary, but because masked prime distracter locations, which are phenomenally invisible, also produce SNP effects (Fitzgeorge et al., 2011). The idea is that event presence which does not reach the level of cognitive awareness cannot have been intentionally processed (Sumner, 2007), indicating that such processing is achieved automatically. Perhaps as a result of this automaticity, this distractor's processing is extensive. The distractor identity and its location are determined, and the location activates its related response, which subsequently undergoes inhibition to prevent its unwanted initiation. A representation of this processing is then stored (Buckolz et al., 2012b; Fitzgeorge \& Buckolz, 2008).

Inhibited responses are deemed to take on an execution resistance (ER) property which acts to discourage their future use, analogous to the residual inhibition proposed by Tipper (2001) but focused on output rather than an unspecified locus. Hence, the distractor response processing sequence includes activation $\rightarrow$ inhibition $\rightarrow$ execution resistance (ER). This ER feature is most readily indicated on free choice trials, where individuals show a significant bias against selecting a former distractor response in favour of choosing its control competitor (within-hand finger options; Fitzgeorge et al., 2011; Lok, 2011). Presumably, the execution resistance property repels efforts to freely select distractor responses. When the later use of the prime distractor response is required (i.e., ignored-repetition trial), one must override distractor response execution resistance before it can be correctly executed. Since execution resistance override takes time to achieve, the ignored-repetition trial response initiation is delayed beyond that of a control trial that lacks an override requirement. The 
result is the SNP effect (RT[ignored-repetition] > RT[Control]), which is deemed to be one of four inhibitory after-effects caused by the execution resistance feature of the prime trial distractor-response (Buckolz et al., 2012b; Stoddart, 2013). According to this scenario, the SNP phenomenon is solely caused by the inhibition of the activated prime distractor response (i.e., an output locus), and is uninfluenced by inhibition that may or may not be associated with the prime distractor's location.

Evidence giving rise to an exclusive output locus for SNP comes from Guy et al. (2006) and Buckolz et al. (2012b). They included many-to-one location-to-response mappings in their SNP procedures. As a result, they were able to generate two trial types whereby the probe target appeared either at the former distractor location or at a previously unoccupied location, but where both required the use of the former distractor response (i.e., ignored-repetition and distractor-response repeat trials, respectively). Reaction time for the distractor response repeat trials were significantly longer than those for ignored-repetition trials, both of which was significantly longer that of control trial latencies. This result shows that using the prime distractor response alone is sufficient to produce significant RT slowing on a probe trial (also see Fitzgeorge et al., 2011, Lok, 2011), and, moreover, that distractor locations may not be inhibited during prime trial processing. This follows from the fact that also using the prime distractor location (ignored-repetition trial) did not increase the RT slowing beyond that seen on distractor-response repeat trials. So, there was no evidence that centrally positioned locations are inhibited in a way that contributes to ignored-repetition delays, and so to SNP (also see Buckolz, Fitzgeorge, \& Knowles, 2012a). In fact, it seems that the re-use of the prime distractor location by the probe target produces a facilitation effect.

The next aspect of the SNP model deals with how the stored representation of primetrial distractor processing is able to participate during probe trial processing in a way that 
generates an SNP effect. At this point, the role of the probe distractor in SNP production is incorporated into the SNP account.

Fitzgeorge and Buckolz (2008) and Buckolz et al. (2012b) posited the existence of two independent neural pathways that retrieved stored distractor processing representations, which were triggered either by the probe trial target or the probe distractor (Schematic 1). In the first instance, when the probe target appears at the prime-trial distractor position, its activation of a former distractor response triggers distractor representation retrieval (i.e., 'response-based retrieval' route, Buckolz et al.). The existence of a response-based retrieval route is indicated by the RT delays found for both ignored-repetition and distractor-response repeat trials (Guy et al., 2006; Buckolz et al.). In the case of distractor response repeat trials, retrieval could not have been triggered because the probe target occupied a former distractor's location, thereby leaving only the response-based retrieval route viable.

A serendipitous yet very instructive finding obtained by Fitzgeorge and Buckolz (2008) pointed to the probe distractor event itself as also triggering prime distractor representation retrieval. In their critical $.25 / .75$ condition, the prime trial contained both a target and a distractor event while the probe trial target also appeared with a distractor $(.25)$ or appeared alone (.75; i.e., target-only trial). The identities of the prime and probe trials fully matched. Fitzgeorge and Buckolz found that the SNP effect was not observed when the probe trial was actually distractor free (i.e., SNP 'removed'), but, surprisingly, SNP was 'restored' when the distractor randomly accompanied the probe target stimulus. Notably, the lack of an SNP effect on distractor-free (target-only) probe trials did not mean that prime-trial distractor processing needed for SNP production had been eliminated. This is because a stored representation of earlier distractor processing was clearly available for SNP restoration (i.e., retrieval) when a probe-trial distractor was present. 


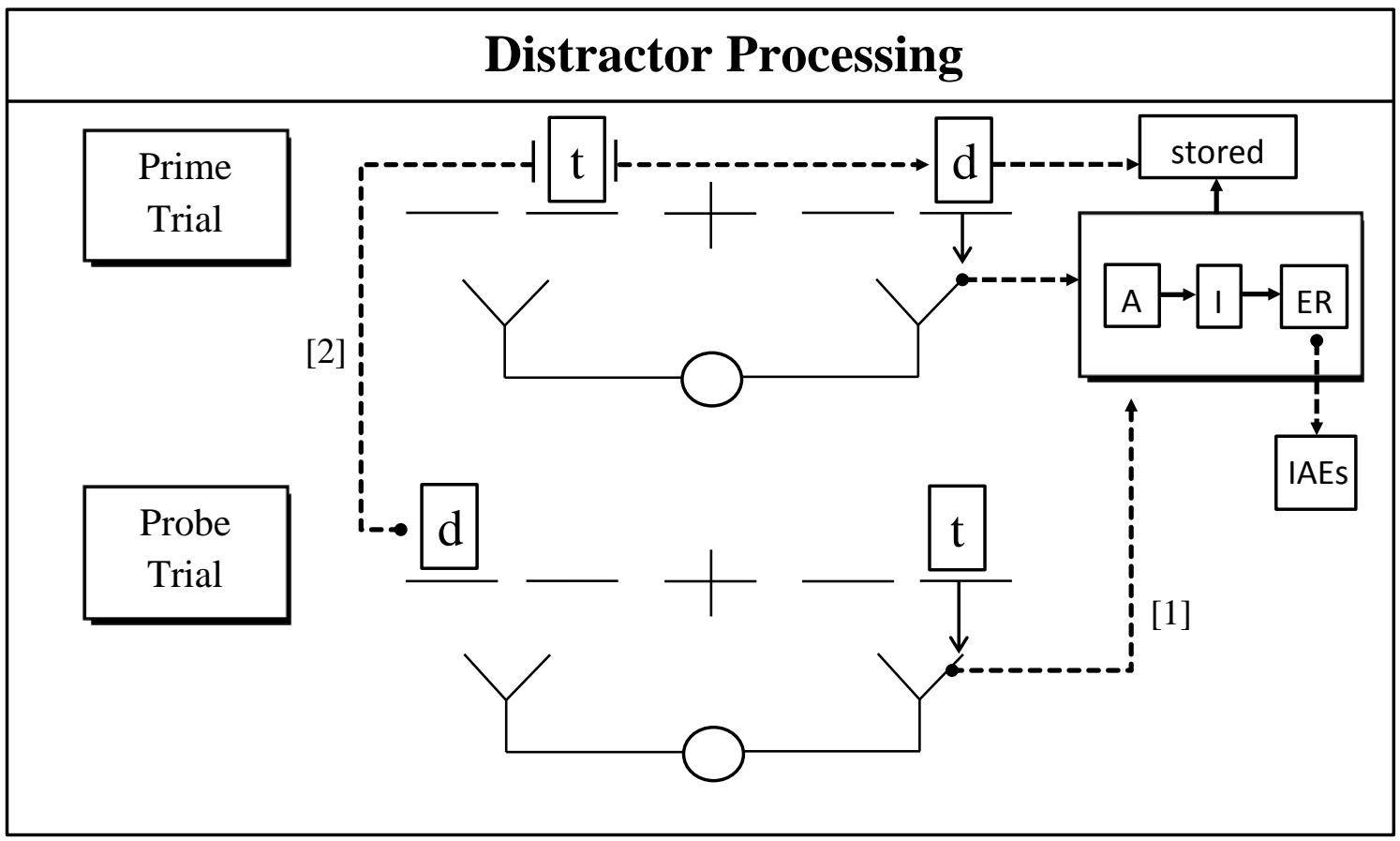

Schematic 1. An illustration of the response-based [1] and the object-based [2] pathways via which stored prime-trial distractor processing representations might be retrieved at the time of probe trial event delivery (Buckolz, Edgar, Kajaste, Lok, \& Khan, 2012). Note. $\mathrm{t}=$ target, $\mathrm{d}=$ distractor, $\mathrm{A}=$ activation, $\mathrm{I}=$ inhibition, $\mathrm{ER}=$ execution resistance, $\mathrm{IAEs}=$ inhibitory after-effects. The prime-probe trial type shown above elicits an ignored repetition trial. 
To accommodate their 'SNP removal' then 'SNP restoration' pattern, Fitzgeorge and Buckolz (2008) concluded that the .25 (distractor present)/.75 (distractor absent) probe trial manipulation motivated the disengagement of the SNP process, in the same way that disengagement occurs when the probe distractor is certain to be absent (e.g., Buckolz et al., 2002; Guy et al., 2004). Furthermore, they proposed that this disengagement is achieved by blocking the retrieval of prime distractor representations. In this instance, it would be accomplished by blocking the 'response-based' route. Most important, the restoration of the SNP effect that accompanied the appearance of a probe distractor event suggested the presence of a second (distractor-triggered) retrieval route (i.e., object-based route; Buckolz et al, 2012), whose retrieved information is capable of generating an SNP effect.

So, overall, when SNP disengagement is motivated, it begins by blocking the response-based retrieval route, which results in SNP absence when the probe is actually distractor-free. When the probe trial contains a distractor, it triggers distractor representation retrieval that circumvents the response-based route block so that an SNP effect is produced (i.e., restored). This would explain why an SNP effect has always been found as long as the probe trial contains a distractor (Fitzgeorge \& Buckolz, 2008). Seemingly, the distractor (object-based) route is not blocked when disengagement is motivated.

Thus, according to the foregoing scenario, the role played by a probe distractor in producing SNP presence/absence is two-staged in nature. In the first stage, a low likelihood of distractor appearance can motivate SNP disengagement, achieved by blocking the response-based route that normally retrieves prime distractor representations that produce SNP during probe-trial processing. This disengagement shows up on distractor-free probe trials. In the second stage, the actual appearance of a probe distractor can restore an SNP effect through its triggering of a retrieval route that captures prime distractor processing representations for probe-trial use. 
It is important to emphasize that the retrieval role posited here for the probe distractor is a direct one, meaning that the probe distractor event accesses stored prime-trial distractor representations without any intermediary influences, which have been previously proposed to explain probe distractor manipulations on negative priming ('contextual similarity' between prime and probe trials; Neill, 1997, or 'inhibitory state' maintenance; Moore, 1994). This point will be taken up again later.

\section{Current Objectives}

The major objective here was to provide support for the view that the probe distractor in location-based tasks has both a motivational (i.e., disengage) and a retrieval (distractor processing representations) role that can influence SNP presence/absence (Fitzgeorge and Buckolz (2008). To do this, we employed the .25 (distractor present)/ .75 (distractor absent) condition utilized by Fitzgeorge and Buckolz. The intent was to motivate the disengagement of the SNP process, revealed by the lack of an SNP effect on distractor-free probe trials, so that we could examine the 'SNP restoration' power of a probe distractor when it was actually present. Regarding the latter, one difference between the current study and that of Fitzgeorge and Buckolz was that the similarity level of the prime and probe distractor identities were varied, ranging from a full match (e.g., Fitzgeorge \& Buckolz, 2008), to a partial match, to a total mismatch. We tested the possibility that if probe distracters do directly retrieve prime trial distractor processing representations, triggering this retrieval may require that the identities of the prime and probe distractor fully match (e.g., Graboi, 1971), or, in the least, partially match. If so, total mismatches between prime and probe distractor identities would fail to trigger retrieval and so would not produce an SNP effect. In short, if SNP restoration was dependent upon prime and probe distractor identities matching to some degree, it would signal a direct retrieval role for probe distracters in location-based tasks. 
A further difference here relative to Fitzgeorge and Buckolz (2008) is that each distractor identity matching level was generated in two distinct ways, using different primeprobe distractor identities (see Table 2, pg. 17). For example, full identity matches were achieved using either a pair of red rectangles or a pair of blue triangles, which appeared on consecutive prime and probe trials (see Table 2). The intent here was to determine whether the impact of the three distractor identity matches levels on SNP restoration depended upon the specific distractor identities used to create these levels. If not, then the SNP restoration data for the probe distractor present trials in this study would more likely generalize to other procedures that differ in this regard.

Finally, on another procedural note, the actual prime-trial and probe-trial distractor identities were fixed for a given subject (but varied between them). In this way, the primeprobe distractor identity matching levels (full, partial, total; along with the identities themselves) were known in advance, simulating Fitzgeorge and Buckolz (2008) in this regard. The motivation for this design feature was that pilot data using the $.25 / .75$ probe distractor presentation likelihood imbalance, where the distractor identity matching levels varied within a trial sequence, had a comparatively low SNP disengagement success rate between subjects (i.e., about 33\%) [See Appendix A]. It may be that varied prime-probe distractor identity matching levels within the same trial sequence interfere with the subjects' perception of the $.25 / .75$ probe-trial distractor occurrence imbalance, and/or interfere with their willingness to disengage SNP. The question here was whether having known distractor identities and known distractor identity similarity levels would improve the disengagement impact of the $.25 / .75$ manipulation 


\section{Method}

\section{Participants}

Sixty university undergraduate students (27 males, 33 females), from the Faculty of Health Sciences, Western University, participated in the study. The age range for the study was $22-41$ years and all participants had normal or corrected-to-normal vision.

\section{Apparatus}

Participants were seated at a desk in a dimly lit room approximately $200 \mathrm{~cm}$ from a 61 $\mathrm{cm}$ (24 inch) computer monitor that contained the visual display for the experiment. For each trial, this display consisted of a white fixation cross (10 $\mathrm{mm}$ wide, $10 \mathrm{~mm}$ high $)$ positioned at the centre of the computer monitor on a black background. This cross was accompanied on either side by two white horizontal bar markers, each measuring $10 \mathrm{~mm}$ horizontally. The bar markers indicated the location on the screen at which the display items (target and/or distractor; $10 \mathrm{~mm}$ wide and $20 \mathrm{~mm}$ high) would appear. All bar markers and the fixation cross were separated from each other by $6 \mathrm{~mm}$, yielding a horizontal display distance of approximately $60 \mathrm{~mm}$ and a horizontal visual angle of about $1.8^{\circ}$.

The four bar marker locations were designated as L1 to L4 from left to right. While seated, each participant places his/her forearms on a desktop that contained a Logitech MK300 wireless keyboard which was stabilized in the middle of the desktop. Keyboard buttons 'D', 'V', 'M' and ' $L$ ' were assigned to locations $\mathrm{L} 1$ through $\mathrm{L} 4$ from left to right. The third digit and index finger on the left hand controlled buttons ' $\mathrm{D}$ ' and ' $\mathrm{V}$ ' respectively, while the same digits on the right hand were used to depress buttons assigned to ' $L$ ' and ' $M$ '. Correctly responding required a button press at its relative location (see Schematic 2) 


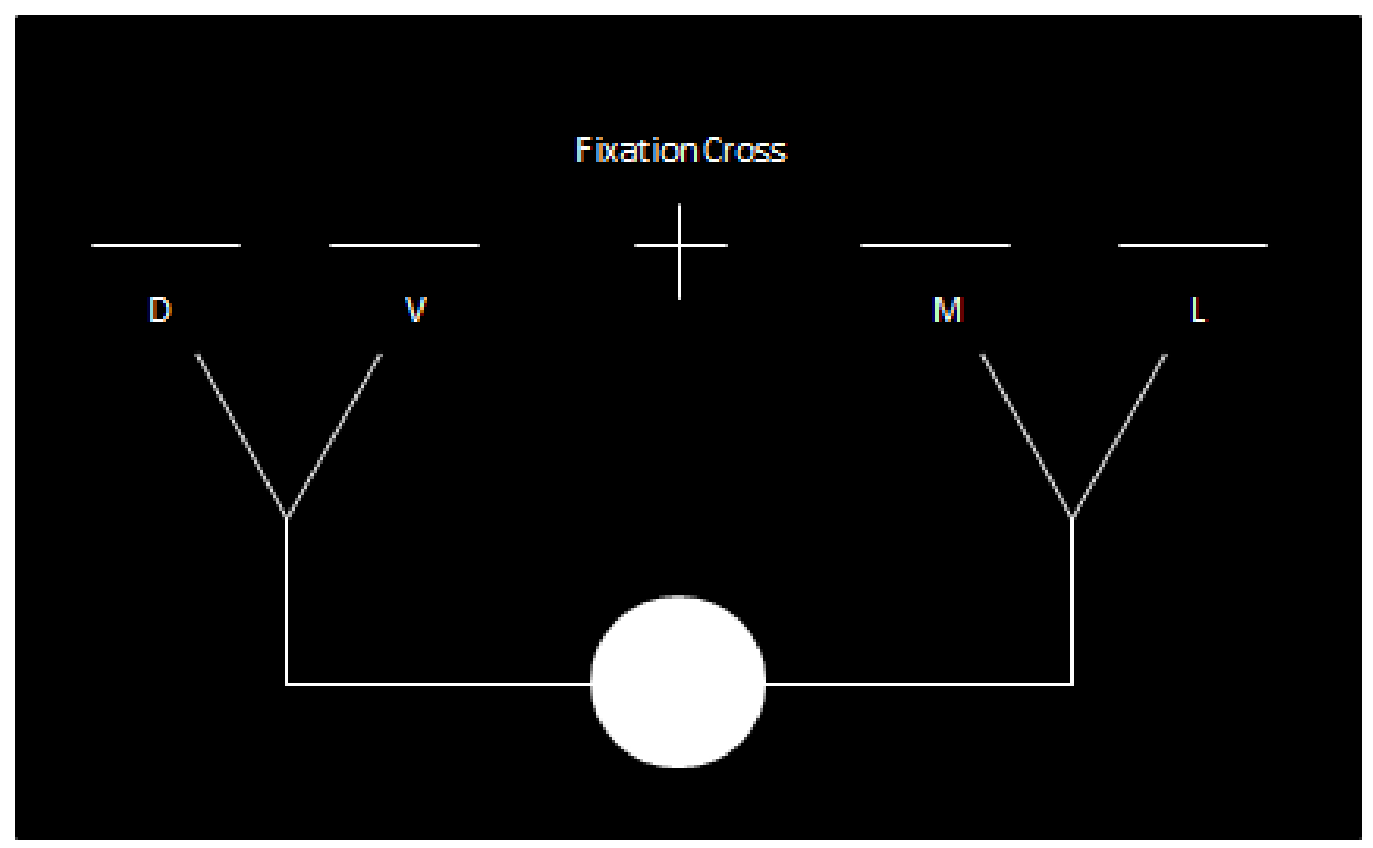

Schematic 2. Screen display of bar markers, fixation cross and stick figure seen by subjects; the letters of the keyboard buttons assigned to each locations are added (DVML) as is "Fixation cross", 


\section{Procedure}

The study had an experimental phase and a preliminary phase whereby subjects engaged in the same task both before and after the experimental component. The testing was completed over a three day period with the first and last day consisting of the target only manipulation and the second day the experimental phase.

\section{Experimental Phase}

In this phase, subjects completed a slightly modified, basic spatial negative priming (SNP) task (Neill et al., 1994), where trials are presented in pairs; first the 'prime', and then the 'probe'. The prime trial always contained a target plus a distractor event, while the probe trial could again hold a target plus a distractor or be distractor-free (i.e., target-only probe), with the probabilities of these two probe trial types being .25 and .75 , respectively.

A trial sequence commenced with a warning tone $(100 \mathrm{~ms})$, whose offset was followed by the location bar markers and the fixation cross for the entire prime trial display (see Schematic 3). The prime trial target and distractor events appeared $200 \mathrm{~ms}$ after tone offset and remained visible for $157 \mathrm{~ms}$ (e.g., Milliken et al., 2000). A correct prime trial target response initiated a probe onset delay of $1000 \mathrm{~ms}$, whose termination coincided with the probe trial presentation, which lasted for $157 \mathrm{~ms}$. The production of the correct probe-trial response initiated an inter-trial delay of $1,500 \mathrm{~ms}$ that ended with the onset of the warning tone, which began the presentation of the next prime-probe trial pair. When an incorrect button-press occurred, the trial sequence halted until the correct response was executed. Button-press error responses were recorded; however, prime-probe trial pairs that contained an error were not used for reaction time analyses. Following the completion of forty trial pairs, a rest was automatically offered. Participation resumed when the spacebar was pressed. 


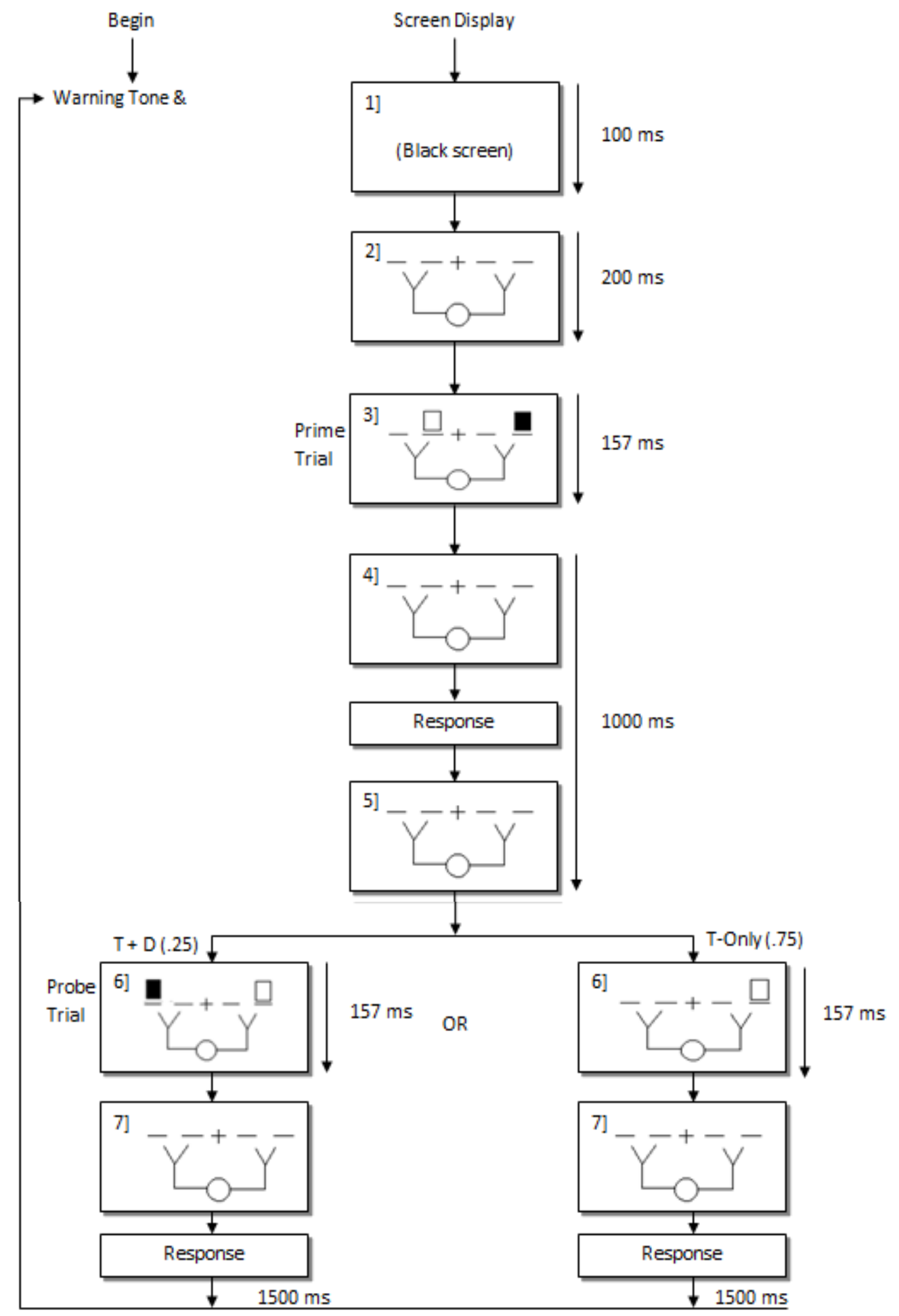

Schematic 3. An illustrative sequence of events for a four-response four locationta sk with a target (white rectangle) plus distractor (black rectangle) prim e trial(Panel 3 ) followed by either a target plus distractor $(T+D)$ or a target-only probe trial(T-Only) (Panels 6). 
When both a target and a distractor event appeared together, they arose randomly and equally often at all possible locations on both prime and probe trials, as was the case when the probe trial contained only a target. This resulted in 144 prime-probe trial pairs where both a target and a distractor were present and 432 prime-probe trial pairs, where the probe held only a target (.25/.75 ratio), for a total of 576 trial pairs. This resulted in 132 ignoredrepetition trials (one location re-used, probe target-to-prime distractor location), 132 targetrepeat trials (one location re-used, probe target-to-prime target location) and 240 control trials (no locations were re-used). The remaining prime-probe event combinations occurred on 72 trials (not used for analysis). The appearance of prime-probe trials was randomized for each trial series so that no participant saw the same order twice.

Subjects were randomly assigned in equal numbers $(n=20)$ to one of three conditions that differed with respect to the degree of similarity between the prime trial and probe trial distractor identities, which either fully matched, partially matched, or totally mismatched each other (i.e., Distractor Identity Similarity Level factor) [see Table 2]. Within each of these three Levels, subjects were again divided randomly and in equal numbers $(n=10)$ into subgroups, with each subgroup having prime and probe trial distractor identities that differed from the other subgroup (i.e., Distractor Identity Subgroup factor). In this way, each subgroup of 10 subjects had their own unique prime trial and probe trial distractor identity pairing, and each of the three Distractor Identity Similarly Levels was generated in two ways, by different distractor identity combinations. For example, in the full match condition, the distractor identity on the prime was a red rectangle followed by a red rectangle on the probe for one subgroup, or a blue triangle distractor followed by a blue triangle for the other subgroup, and so on (Table 2). Accordingly, subjects knew throughout the actual prime and probe trial distractor identities they would encounter and hence how their paired distractor 


\section{Table 2}

Distractor identity matching levels (full match, partial match, total mismatch: betweensubjects), each produced in two ways by using different prime and probe trial distractor identities (between-subjects: Subgroups).

Distractor Identity

Matching Level

Full Match ${ }^{\mathrm{a}}$ :

Partial Match ${ }^{\mathrm{a}}$ :

Total Mismatch ${ }^{\mathrm{a}}$ :
Subgroups

${ }^{\mathrm{b}} \mathrm{Red}$ rectangle $\longrightarrow$ Red rectangle

Blue triangle

${ }^{\mathrm{b}}$ Red rectangle $\longrightarrow$ Red triangle

${ }^{\mathrm{b}}$ Blue triangle $\longrightarrow$ Blue rectangle

${ }^{\mathrm{b}}$ Red rectangle $\longrightarrow$ Yellow cross

a 20 participants within each condition but only experienced one type of prime-probe distractor identity matching level. ${ }^{\mathrm{b}} 10$ subjects per distractor identity combination. 
identities would match up. Thus, both of the aforementioned factors were between-subject manipulations.

\section{Preliminary Phase}

The prime trials of the SNP task completed in this phase contained both a target and a distractor event, whose placements were governed as in the Experimental Phase. In contrast, the probe trial predictably $(100 \%)$ held only a target, which appeared randomly and equally often at all four locations. This resulted in 192 prime-probe pairs; 48 ignored repetition, 48 target-repeat trials, and 96 control trials. The target and distractor identities used in this task were the same for all subjects, with the task being completed once before and once after the experimental phase.

The information/instructions given to the subjects prior to the start of testing in each phase of the study were as follows: (a) they were to respond as quickly as possible while maintaining accuracy, using the finger response assigned to the location occupied by the target event, and to ignore a distractor event should one be present, (b) trials would be presented in pairs beginning shortly after a warning tone, with the second trial timed to appear shortly after a correct response. The time between successive trial pairs would be noticeably longer than other time delays experienced and would again begin with the sounding of the warning tone, (c) the probe trial would always contain only a target (Preliminary Phase), and (d) the proportion of probe distractor present (.25) and probe distractor absent (.75) trials was indicated, as was the fact that the distractor identities on prime and probe trials would be the same throughout their participation (Experimental Phase). 
Subjects completed a practice session of approximately 10-15 trial pairs (Experimental Phase task) and were afforded the opportunity to ask question, in order to ensure they understood the task demands. Subjects then completed the pre-experimental component of the Preliminary Phase, which took approximately 20 minutes. On the following day, subjects undertook the Experimental Phase which lasted between 30-40 minutes. The post-experimental aspect of the Preliminary Phase was then completed the following day.

Reaction times of less than $100 \mathrm{~ms}$ (anticipations) or greater than $1000 \mathrm{~ms}$ (insufficient vigilance), along with any prime-probe trial pair that involved a button-press error, were excluded from the reaction time analyses. All ANOVA data in the report are assumed to be independent and that errors were normally distributed (see Guy et al., 2006). 


\section{Results}

Preliminary Phase: $100 \%$ Distractor-free Probe Trials, Target + Distractor Prime Trials

Both before and after their participation in the experimental portion of our study, all individuals undertook an SNP procedure where the probe always lacked a distractor. In both cases, the SNP effect was eliminated: before, $t(59)=.304, \underline{p}=0.763, S E=3.67$ and after, $t(59)=.321, \underline{p}=0.750, S E=3.22($ Table 3$)$. These results have a procedural implication noted later.

\section{The Experimental Phase}

\section{Distractor-occupied Prime Trial Locations: Inhibitory After-effects}

Recall that the Distractor Identity Similarity Levels (full match, partial match, total mismatch), and the actual prime-to-probe distractor identities used to produce these levels, were between-subjects factors in this study (Table 2). Each of the Identity Similarity levels was produced in two ways, each using distinct prime-probe distractor identities. For example: a full match level of similarity occurred when a red rectangle was followed by a red rectangle, or a blue triangle followed a blue triangle; while a partial match occurred when a red rectangle was followed by a red triangle, or when a blue triangle was followed by a blue rectangle (shape mismatches).

The first analyses of variance (ANOVAs) series conducted aimed to determine whether the particular identities used to create each of the three Distractor Identity Similarity levels mattered, in that they influenced the pattern of results produced by these levels on the 'SNP restoration' data. If not, then outcomes of the data produced by the Identity Similarity factor would more likely be generalizable other work using different distractor identities than those used in this investigation. 
Table 3

Mean reaction times (ms) for t-only probes (100\%); pre-and-post experimental task.

\begin{tabular}{lcc}
\hline & \multicolumn{2}{c}{ Preliminary phase } \\
\cline { 2 - 3 } & \multirow{2}{*}{ Pre } & Post \\
\cline { 2 - 3 } $\begin{array}{lc}\text { Probe } \\
\text { Configuration }\end{array}$ & T-only & \\
\hline Trial-type & & T-only \\
Ignored-repetition & $444(6.3)$ & $419(4.7)$ \\
& {$[2.2]$} & {$[2.4]$} \\
Control & $443(6.2)$ & $417(5.3)$ \\
& {$[2.0]$} & {$[2.2]$} \\
Target-repeat & $417(5.7)$ & $398(4.1)$ \\
& {$[2.6]$} & {$[2.0]$} \\
\hline Spatial Negative Priming & 01 & 02 \\
Target-repeat Effect & $-26^{*}$ & $-21^{*}$ \\
\hline
\end{tabular}

Note. Spatial Negative Priming = Ignored-repetition - Control; Target-repeat Effect $=$ Targetrepeat - Control. ()$=$ standard error $(\mathrm{ms}) ;[]=$ button press error percentage. ${ }^{*} p<0.05 . n=60$ 


\section{Probe-trial Reaction Times}

Three ANOVAs were calculated using subjects mean RTs, one for each level of the Distractor Identity Similarity Level factor, Probe Content (target + distractor, target-only), Probe-trial Type (ignored-repetition, control), and Distractor Identity Pairings Subgroup (Table 2) as the main factors.

When the prime-probe identities fully matched, neither the Distractor Identity Subgroup main effect, $F(1,18)=1.06, \underline{p}=.316, M S E=6472$, nor its interaction with the other two factors, $F \mathrm{~s}<1$, were significant. As expected, probe trial reaction times were significantly longer for target + distractor $(440 \mathrm{~ms})$ than for target-only $(417 \mathrm{~ms})$ probes, $F(1,18)=46.407, \underline{p}<0.01, M S E=221.47$, and for ignored-repetition $(434 \mathrm{~ms})$ than for control (424 ms) trials, revealing the classic SNP effect, $F(1,18)=19.41, \underline{p}<0.01, M S E=104.64$. However, the fact that Probe Trial Type did not interact with the other factors, singly or together, $F \mathrm{~s}<1$ (except for the Probe Trial Type x Distractor Identity Subgroup interaction, $F(1,18)=1.44, \underline{p}=.245, M S E=106.64)$, was not anticipated, specifically regarding the fact than an SNP effect was present for target-only probe trials.

This same ANOVA result pattern occurred for the partial match, where the Subgroup main effect $(F(1,18)=0.96, \underline{p}=.340, M S E=8144)$ nor the interaction $(F \mathrm{~s}<1)$ term was significant. Similarly, the total mismatch distractor identity did not demonstrate a main effect for the subgroups $(F(1,18)=1.77, \underline{p}=0.20, M S E=3937)$ nor an interaction with the other two factors $(F \mathrm{~s}<1)$. Collectively, these analyses that indicate that the manner in which the various Distractor Identity Similarity Levels were produced did not influence the impact produced by the remaining two factors. Hence, this manipulation was discarded from further analyses that tested the influence of the distractor identity similarity levels directly in the same ANOVA. 


\section{Table 4}

Mean reaction times $(R T, m s)$ for the Trial Type (ignored-repetition, control, target-repeat) and Distractor Identity Matching levels (full match, partial match, total mismatch) factors.

Distractor Identity Matching Levels

\begin{tabular}{|c|c|c|c|c|c|c|}
\hline \multirow{2}{*}{$\begin{array}{l}\text { Probe } \\
\text { Configuration }\end{array}$} & \multicolumn{2}{|c|}{ Full Match } & \multicolumn{2}{|c|}{ Partial Match } & \multicolumn{2}{|c|}{ Total mismatch } \\
\hline & $T+D$ & T-only & $T+D$ & T-only & $T+D$ & T-only \\
\hline \multicolumn{7}{|l|}{ Trial-type } \\
\hline Ignored-repetition & $\begin{array}{c}446(10.1) \\
{[3.2]}\end{array}$ & $\begin{array}{c}423(8.6) \\
{[4.1]}\end{array}$ & $\begin{array}{c}473(12.6) \\
{[3.7]}\end{array}$ & $\begin{array}{l}442(10.4) \\
{[4.0]}\end{array}$ & $\begin{array}{c}455(9.9) \\
{[4.4]}\end{array}$ & $\begin{array}{c}427(6.7) \\
{[5.6]}\end{array}$ \\
\hline Control & $\begin{array}{c}435(9.2) \\
{[2.5]}\end{array}$ & $\begin{array}{c}412(9.2) \\
{[2.2]}\end{array}$ & $\begin{array}{c}459(9.1) \\
{[2.3]}\end{array}$ & $\begin{array}{c}436(9.4) \\
{[2.1]}\end{array}$ & $\begin{array}{c}445(7.9) \\
{[2.2]}\end{array}$ & $\begin{array}{c}4156.3) \\
{[2.6]}\end{array}$ \\
\hline Target-repeat & $\begin{array}{c}428(7.4) \\
{[3.6]}\end{array}$ & $\begin{array}{c}401(6.7) \\
{[3.2]}\end{array}$ & $\begin{array}{c}454(7.5) \\
{[4.1]}\end{array}$ & $\begin{array}{c}412(8.3) \\
{[4.8]}\end{array}$ & $\begin{array}{c}441(6.8) \\
{[2.2]}\end{array}$ & $\begin{array}{c}405(5.0) \\
{[5.8]}\end{array}$ \\
\hline $\begin{array}{l}\text { Spatial Negative Prir } \\
\text { Target-repeat Effect }\end{array}$ & $\begin{array}{l}\text { ing } 11 * \\
-07 *\end{array}$ & $\begin{array}{l}11^{*} \\
-11^{*}\end{array}$ & $\begin{array}{l}14^{*} \\
-05\end{array}$ & $\begin{array}{c}06 \\
-24 *\end{array}$ & $\begin{array}{l}10 * \\
-04\end{array}$ & $\begin{array}{r}12^{*} \\
-10^{*}\end{array}$ \\
\hline
\end{tabular}

Note. Spatial Negative Priming = Ignored-repetition - Control; Target-repeat Effect $=$ Targetrepeat - Control. $\left(\mathrm{)}=\right.$ standard error $(\mathrm{ms}) ;[]=$ button press error percentage. ${ }^{*} p<0.05 . n=60$ 
Accordingly, we calculated an ANOVA using mean subject RTs with Distractor Identity Similarity level (full match, partial match, total mismatch), Probe Trial Type (ignored-repetition, control) and Probe Trial Content (target + distractor, target-only) as the main factors. The Probe Trial Type factor yielded a significant SNP effect $(10 \mathrm{~ms}), F(1,57)=$ $27.89, \underline{p}<0.01, M S E=227.16$; however, more notably, this factor did not interact with the other factors, singly or in combination, $F \mathrm{~s}<1$. The SNP effect was present on target + distractor probe trials when the prime-probe identities fully matched $(11 \mathrm{~ms})$, partially matched $(14 \mathrm{~ms})$ or totally mismatched $(10 \mathrm{~ms})$. The same held when the probe trial was distractor free (Table 4). Thus, unlike Fitzgeorge and Buckolz (2008), the .25 (distractor present)/ .75 (distractor absent) probe distractor manipulation did not motivate the disengagement of the SNP process for a sufficient number of subjects to see an overall SNP disengagement effect. Hence, it was not possible at this point to examine the main issue here, which was to test SNP restorative impact of the probe distractor as a function of its similarity level to the prime distractor identity.

Given the SNP disengagement success of the $.25 / .75$ probe distractor manipulation in past work (Fitzgeorge \& Buckolz, 2008; Fitzgeorge, 2009), an obvious possibility for its failure to do so in this study is that the SNP disengagement motivation influence of the $.25 / .75$ manipulation is subject specific. Accordingly, the target-only probe trial data was examined for each subject. Using an SNP criterion value of $5 \mathrm{~ms}$ or less (2 times the standard error of the mean SNP value for all subjects), we were able to place 10 subjects in each of the fully match, partial match, and total mismatch conditions. A preliminary t-test looking at the RT difference between ignored-repetition and control trials (i.e., $\mathrm{SNP}=1.2 \mathrm{~ms}$ ) for the targetonly probe trials for these 30 individuals, was non-significant, $t(29)=1.69, \underline{p}=.102, S E=0.69$. Accordingly, the $.25 / .75$ manipulation was deemed to have successfully motivated these subjects to disengage the SNP process (Table 5 also see Figure 2). 
Table 5

Mean reaction times $(R T, m s)$ for the Trial Type (ignored-repetition, control, target-repeat) and Distractor Identity Matching levels (full match, partial match, total mismatch) factors.

Distractor Identity Matching Levels

\begin{tabular}{|c|c|c|c|c|c|c|}
\hline \multirow{2}{*}{$\begin{array}{l}\text { Probe } \\
\text { Configuration }\end{array}$} & \multicolumn{2}{|c|}{ Full Match } & \multicolumn{2}{|c|}{ Partial Match } & \multicolumn{2}{|c|}{ Total mismatch } \\
\hline & $T+D$ & T-only & $T+D$ & T-only & $T+D$ & T-only \\
\hline \multicolumn{7}{|l|}{ Trial-type } \\
\hline Ignored-repetition & $\begin{array}{c}469(12.8) \\
{[3.5]}\end{array}$ & $\begin{array}{l}438(10.1) \\
{[3.9]}\end{array}$ & $\begin{array}{l}454(12.8) \\
{[4.1]}\end{array}$ & $\begin{array}{l}422(10.1) \\
{[4.0]}\end{array}$ & $\begin{array}{c}443(12.8) \\
{[4.1]}\end{array}$ & $\begin{array}{l}413(10.1) \\
{[4.6]}\end{array}$ \\
\hline Control & $\begin{array}{c}455(11.1) \\
{[2.0]}\end{array}$ & $\begin{array}{c}434(10.1) \\
{[2.2]}\end{array}$ & $\begin{array}{l}445(11.1) \\
{[2.8]}\end{array}$ & $\begin{array}{l}422(10.1) \\
{[2.6]}\end{array}$ & $\begin{array}{c}442(11.1) \\
{[2.9]}\end{array}$ & $\begin{array}{c}413(10.1) \\
{[2.7]}\end{array}$ \\
\hline Target-repeat & $\begin{array}{c}436(9.5) \\
{[3.2]}\end{array}$ & $\begin{array}{c}412(8.7) \\
{[2.9]}\end{array}$ & $\begin{array}{c}449(9.6) \\
{[4.5]}\end{array}$ & $\begin{array}{l}401(8.7) \\
{[4.0]}\end{array}$ & $\begin{array}{c}432(9.6) \\
{[3.0]}\end{array}$ & $\begin{array}{c}396(8.7) \\
{[3.2]}\end{array}$ \\
\hline $\begin{array}{l}\text { Spatial Negative Prir } \\
\text { Target-repeat Effect }\end{array}$ & $\begin{array}{l}\text { Ing } 14 * \\
-19 *\end{array}$ & $\begin{array}{l}04 \\
-22 *\end{array}$ & $\begin{array}{l}09 \\
04\end{array}$ & $\begin{array}{c}00 \\
-21^{*}\end{array}$ & $\begin{array}{r}01 \\
-10\end{array}$ & $\begin{array}{c}00 \\
-17^{*}\end{array}$ \\
\hline
\end{tabular}

Note . Spatial Negative Priming = Ignored-repetition - Control; Target-repeat Effect $=$ Target repeat - Control. $(\mathrm{)})$ standard error $(\mathrm{ms}) ;[]=$ button press error percentage. $* p<0.05 . n$ $=30$ 
Looking at SNP restoration on the target + distractor probe trials (see Figure 1), a significant SNP effect $(14 \mathrm{~ms})$ was observed when the prime and probe trial distractor identities fully matched, $t(9)=2.407, p=0.039, S E=5.75$, but not when distractor identities partially matched, $t(9)=0.093, p=0.928, S E=5.58$, or totally mismatched, $t(9)=1.74, p=$ $0.116, S E=5.13($ Table 5)

Target-occupied Prime Trial Locations: After-effects

Here, we tested the after-effects associated with a target-occupied prime trial location when the probe target later re-appeared at this same location (i.e., a target-repeat trial). An ANOVA was calculated using mean within-subject reaction times, and with Trial Type (target-repeat, control), Distractor Identity Similarity Level (full match, partial match, total mismatch) and Probe Trial Type (target + distractor, target-only) [Table 5]. The three-way interaction was significant, $F(2,27)=3.50, \underline{p}<0.05, M S E=104$. Related post-hoc tests revealed that target-repeat trials produced a reliable reduction in probe target reaction time (beneficial after-effect) for the target-only probe trials and when, on distractor present trials, the prime and probe trial distractor identities fully matched (Table 5: Buckolz et al., 2008; Fitzgeorge \& Buckolz, 2008). 


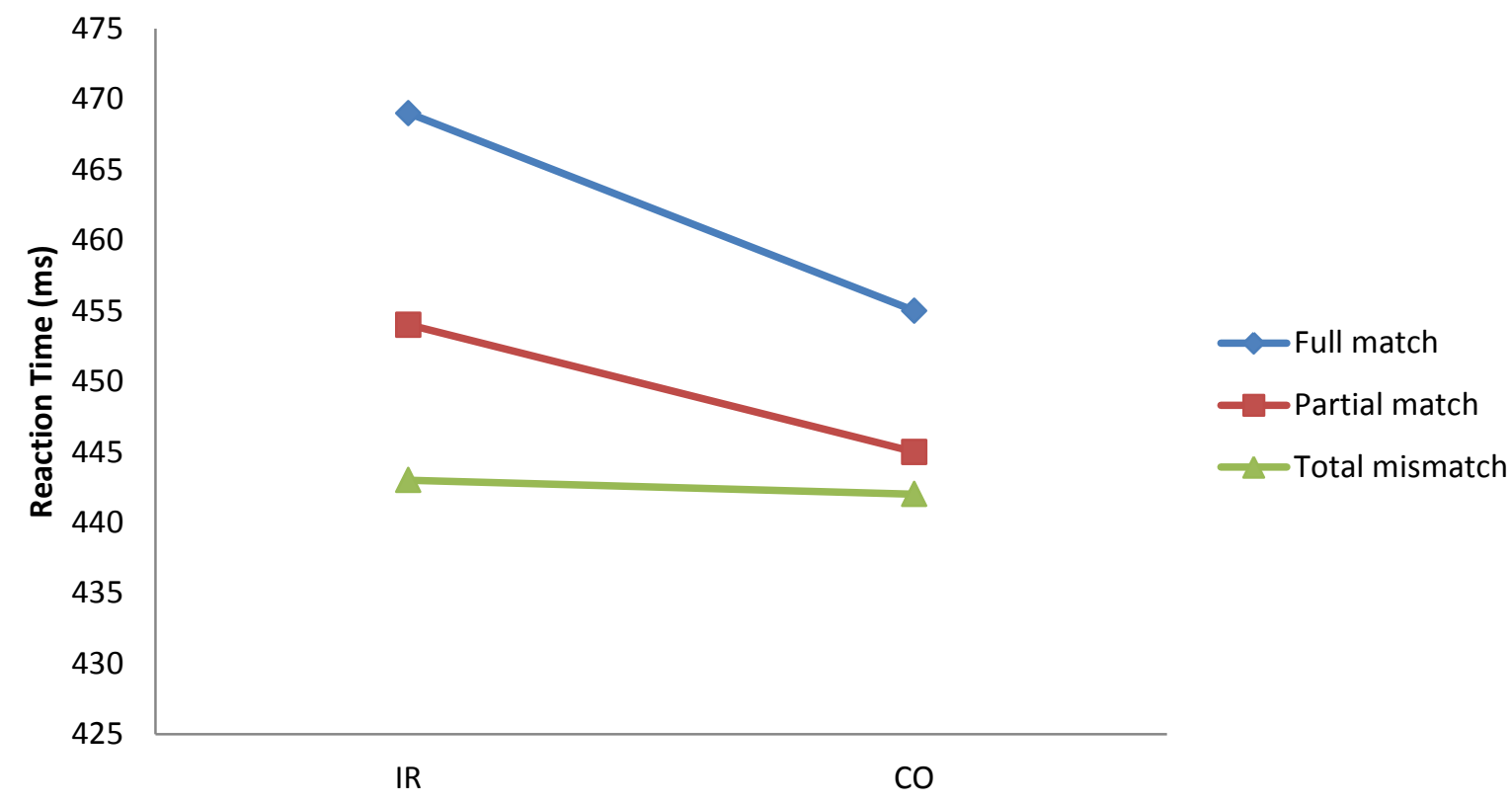

Figure 1. Mean Probe-trial reaction times as a function of Distractor Identity Similarity levels (full match, partial match, total mismatch), Probe-type (ignored repetition [IR] vs. control [CO]) for target-plus-distractor probe trials. $N=30$ 


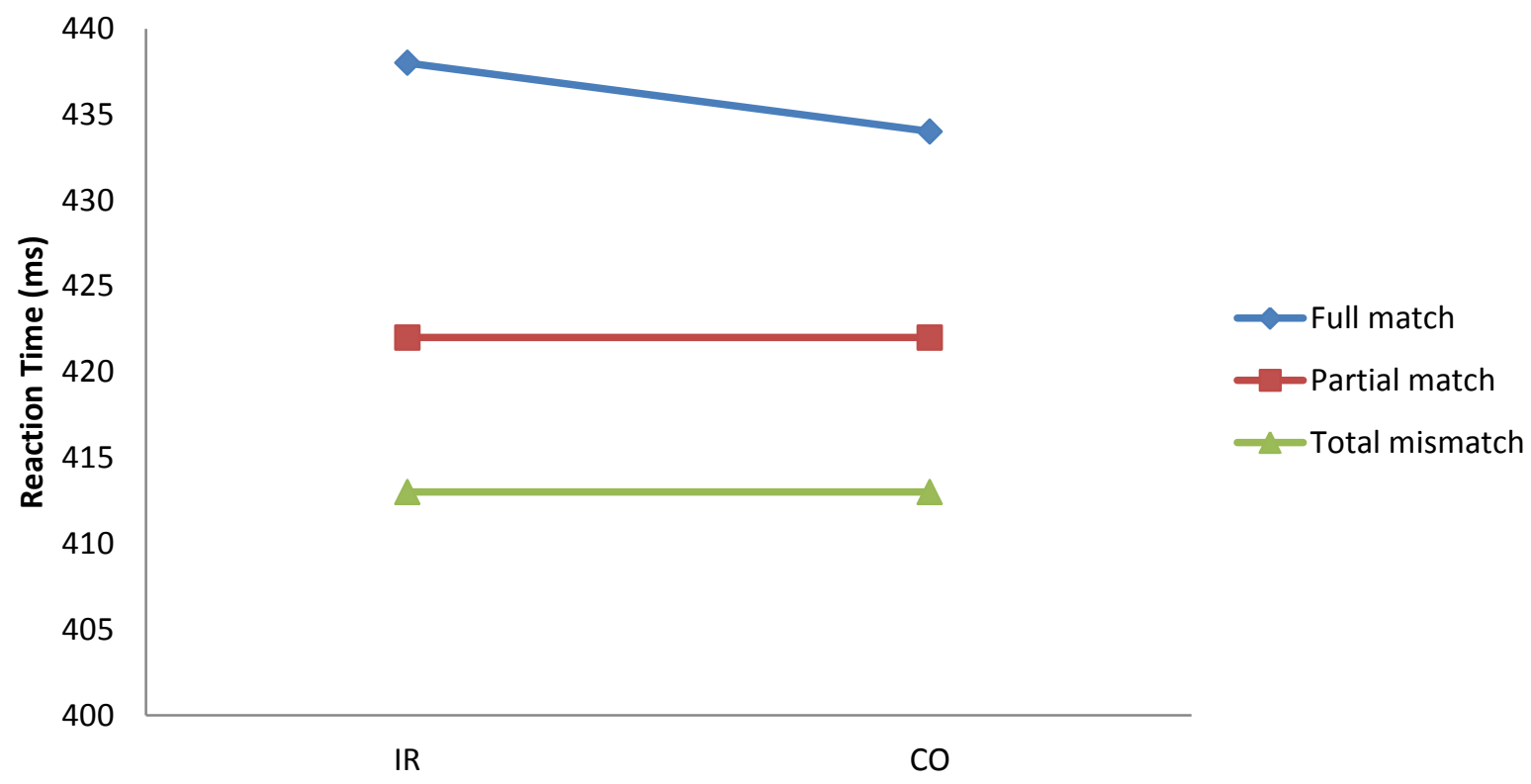

Figure 2. Mean Probe-trial reaction times as a function of Distractor Identity Similarity levels (full match, partial match, total mismatch), Probe-type (ignored repetition [IR] vs. control [CO]) for target-only probe trials. $N=30$ 


\section{Discussion}

The global aim in this study was to better understand the role played by a probe distractor in SNP production/absence in a location-based, spatial negative priming (SNP) task. We hoped to reinforce the view that the probe distractor can have both a 'motivational' (disengagement) and a 'retrieval' (distractor response representation) influence when appropriately manipulated (Fitzgeorge \& Buckolz, 2008). More particularly, we looked to see whether the presence of a probe distractor event would restore an SNP effect (retrieval role) that was otherwise absent (i.e., disengaged motivational role), and to determine whether any SNP restoration depended upon the degree to which the prime and probe distractor identities matched (i.e., full match, partial match, total mismatch).

Testing the SNP remove/restoration impact of a probe distractor naturally first requires that the SNP phenomenon be removed or prevented, which is indicated by the lack of an SNP effect on distractor-free probe trials. We utilized a .25 (probe distractor present)/.75 (probe distractor absent) probability imbalance to motivate SNP removal, and instituted two procedures aimed at enhancing the effectiveness of the SNP removal impact of the $.25 / .75$ procedure on individual subjects. First, we wanted some assurance that the subjects to be used in this experiment were able to prevent SNP production, and that they would do so because of a probe-trial distractor manipulation. Accordingly, we had subjects undertake a series of trials where the probe distractor was predictably absent (i.e., target-only probe trial), a manipulation that has consistently eliminated the SNP effect in past work using target plus distractor prime trials, as we did here (e.g., Buckolz et al., 2002; Guy et al., 2004).

The second procedural variation involved having the prime and the probe distractor identities fixed for a given group of individuals, although they varied between subjects. In this way, not only were the distractor identities themselves known in advance, the type of 
distractor identity matching level was also known, thereby simulating the certainty aspect of Fitzgeorge and Buckolz (2008). We deemed it possible that their success with the $.25 / .75$ procedure in terms of preventing SNP might have evolved because of the certainty associated with the prime-probe distractor relationships, possibly because this certainty elevated the clarity of the $.25 / .75$ probe trial distractor likelihood presence to the individual.

Before turning to the impact of these two particular procedural variations, we first want to note that there was another procedural manipulation undertaken here whereby each of the three distractor identity matching levels was produced in two ways, by using different prime-probe distractor identities. The idea was to see whether the actual distractor identities used to create these levels caused these levels to have different effects on the SNP removal and restoration data. This was not the case, indicating that the results obtained here should generalize to other procedures utilizing distractor identities other than those employed in this study. Additionally, of course, this factor was not utilized in subsequent analyses.

The $100 \%$ distractor-free probe trial procedure was successful overall in eliminating the SNP effect in our subjects. Together with past work (e.g., Buckolz et al., 2002; Guy et al., 2004), this result shows that this probe distractor condition is a consistent means of motivating individuals to disengage the SNP process, and it also indicates that our subjects were able and willing to do so on the basis of a probe trial distractor manipulation.

Consequently, the marginal success rate of the $.25 / .75$ procedure here in preventing SNP (about $50 \%$ of the subjects) could not be attributed to the fact that subjects were incapable of SNP prevention, or to the fact that they were not capable of being influenced by a probe distractor manipulation influenced by the probe distractor probability manipulation (i.e., distractor absence). Furthermore, having the prime and probe trial identities fixed, along with the distractor identity matching condition, did little to enhance SNP removal rate for the 
$.25 / .75$ procedure (i.e., pilot study, removal was about $33 \%$ of subjects). It will be important in the future to see whether other procedures can be used that would enhance the disengagement influence of a probe-trial distractor present/absence imbalance. For now, the important procedural point indicated by the results in this study and in the pilot study (see Appendix A), which was not evident from the Fitzgeorge and Buckolz (2008) report, is that the SNP disengagement success achieved by the $.25 / .75$ probe-trial distractor frequency manipulation is variable and relatively low between samples of subjects. Further study of the 'SNP restore' impact of a probe trial distractor should bear this in mind and to design their study accordingly. It might also be advisable to test whether an even greater probability imbalance between probe distractor present and probe distractor absent trials (e.g., .15/.85) is more consistent in producing SNP removal, in spite of the fact that, if successful, more trials would be required due to the smaller number of the target + distractor probe opportunities to test the SNP restore question.

With the subjects deemed to have disengaged the SNP process in the $.25 / .75$ experimental condition $(n=30)$, we were able to test the 'SNP restore' impact of the presence of a probe distractor. We learned that SNP was restored by a probe distractor as long as its identity fully matched that of the prime distractor; partial or total distractor identity mismatches did not yield SNP effects (Table 4). There are two straightforward implications of this finding.

\section{A Retrieval Role for the Probe Distractor in a Location-based Task}

The first implication is that the dependency of SNP restoration upon distractor identity similarity is consistent with a retrieval role for the probe distractor, thereby supporting prior work on this account (Fitzgeorge \& Buckolz, 2008). As envisioned by Fitzgeorge and Buckolz, and later in more detail by Buckolz et al. (2012b), the normally used 
'response-based' retrieval route (Schematic 1) is blocked in response to the $.25 / .75$ distractor present/absent manipulation, evidenced by the absence of SNP on distractor-free probe trials (Buckolz et al.). The probe distractor (object-based route) can circumvent this blockage by triggering its own representation retrieval, and so restore an SNP effect. What we have learned here is that this retrieval is not inevitable, taking place only when the prime and probe distractor identities fully match.

Quite by chance, the target-repeat data here (Table 5), when considered along with the SNP findings above, allows us to comment on the format in which prime-trial distractor processing representations are stored. This matter is important to address in the current context, not only because the data are novel, but because storage format can affect, and so help us understand, the role played by a probe distractor in producing an SNP effect. Briefly put, the data indicate that representations of prime trial processing in location-based tasks are stored in an episodic manner (e.g., Neill, 1997; Neill, 2007), where event identities (target and distractor), but not event numbers, are integral to representation retrieval.

To explain, Neill, Valdes, Terry, and Gorfein (1992; Episodic Retrieval Theory) some time ago proposed that the storage of past (prime trial) experiences is done in an episodic manner, the defining feature of which is the inclusion of task-irrelevant information unrelated to successful performance (i.e., distractor event, contextual aspects), along with task-relevant material (i.e., target stimulus). In negative priming tasks, the probe trial would serve as a retrieval cue for accessing stored prime trial representations. Appropriate changes to the contextual similarity between the prime and probe trial, such as those brought about by probe trial distractor manipulations, could obviate episode retrieval, thereby eliminating the negative priming effect. 
According to this view, prime target and prime distractor event processing representations would be part of the same stored episode, predicting that a failure to retrieve one event's representation should be accompanied by a failure to retrieve the representation of other event in the episode (this is exactly what we found here). When the identities of the distractors on successive prime-probe trials partially or fully mismatched, SNP failed to be restored, showing that prime distractor processing representations had not taken place. Under these same distractor identity matching levels, target-repeat trials failed to produce a beneficial latency after-effect observed in the full matching level (Table 5), indicating that the retrieval of prime-trial target processing had again not occurred. These results are certainly consistent with the idea that the prime event representations were stored in a common episode. The fact that changing distractor identity from prime to probe trials caused retrieval prevention (SNP and target-repeat facilitation effects absent) indicates that event identities are an important part of stored episodes, even in location-based tasks.

What is not part of the stored episode in location-based tasks is the number of events in the episode, or, at least, number matching is not a retrieval pre-requisite. After-effects occur when the event numbers mismatch and can be absent when they match. Looking at the former, when prime-probe event numbers mismatched here with the target-only probe trials, target-repeat trials nonetheless produced significant reaction facilitation (i.e., retrieval). Notably, this result indicates that it does not matter how many events appear on the probe (relative to the prime), only that whatever event does appear, it must not mismatch events stored in the episode if retrieval is to occur. The unimportance of event number mismatches to retrieval in location-based tasks was also evident in past work, but via the SNP effect, which was significant when prime-probe event numbers differed (e.g., Buckolz et al., 2008; Guy et al., 2004; Neill et al., 1994). Mistakenly, this result has been used at times to claim 
that prime trial processing representations in location-based tasks are not stored episodically (e.g., Buckolz et al.).

It follows from the above that the lack of an SNP effect seen here (Table 3) and elsewhere (Guy et al., 2004) with the certain absence of the probe distractor, following a target + distractor prime, is not the result of different prime-probe event numbers. Rather, as we have suggested here, the manipulation of probe trial distractor probability, including its certain or likely (.25/.75 condition) absence, is better characterized as influencing the motivation to disengage the SNP process via a retrieval block (Buckolz et al., 2012b).

Turning now to when prime-probe event numbers matched here $(\mathrm{T}+\mathrm{D}$ probe trials), the SNP effect, and hence retrieval, were lacking when the prime and probe distracter identities did not fully match. Again, event identity matching, rather than event number matching, is the critical retrieval factor in our location task.

\section{The Role of the Probe Distractor in Maintaining an Inhibitory State}

The second implication of the restore SNP data in this study is that it does not support another proposed role for a probe distractor, one that does not explicitly see it in a retrieval role (Moore, 1994). Relying largely upon results from the identity variant of the negative priming task, Moore learned that a probe distractor was associated with the appearance of identity negative priming (NP) when it actually generated a probe response that competed with the required target output (i.e., a 'conflict' distractor), or when it was difficult to ascertain whether the probe distractor was of the conflict type or not. Moore then suggested that conflict probe distracters, threatened (or appeared to threaten) correct probe-trial response selection because they activated an incorrect alternative (i.e., essentially an 'error protection' effect). Presumably, an inhibitory state helps with incorrect response inhibition. This much seems more or less understandable. 
What needs clarification in Moore's (1994) account is why a maintained 'inhibitory state' should also cause the presence of an 'identity NP' effect (i.e., the latter is used to detect the presence of the former). This is because the processes that underlie the identity negative priming effect are in one instance 'deleterious' (i.e., they delay target processing on ignoredrepetition trials), and, in the other case, are actually 'unhelpful' with disposing of the incorrectly activated probe response; presumably the reason that these inhibitory after-effects were kept active in the first place. This is especially so in identity NP tasks, where prime and probe distractor identities mismatch. Hence, the inhibited prime distractor response is different from the response that would be activated by a probe distractor on a control trial; consequently, the former could not assist in dealing with the latter. So, it seems that proposing the maintenance of an inhibitory state role for conflict probe distractors, even in identity-based tasks, has some logical gaps that need addressing. Consequently, the lack of support for this probe distractor role in this study is less disconcerting.

All of the probe distractor-present trials were of the response conflict type in the current findings. According to Moore (1994), an SNP effect should have been restored on these trials, irrespective of prime-probe distractor identity similarity. This was not the case, of course. Spatial negative priming was absent when the prime and probe trial distractor identities did not match, in spite of the fact that the presence of a distractor was a threat to probe-trial response selection accuracy. So, retrieval of prime-trial distractor processing representations needed to produce SNP, was not triggered by a response conflict. So, as it was suggested earlier, the connection between the presence of a probe-trial distractor and SNP production is one of retrieval (of the prime-trial distractor processing representations). There is no need to surmise that its ability to cause a probe-trial response conflict is a factor in this relationship. 


\section{General Discussion}

\section{Spatial versus Identity Negative Priming Tasks: Probe Distractor Processing}

There is some interest in determining whether the processing underlying the identity and location negative priming (NP) effects are comparable (e.g., Chao, 2011). One way to contribute to answering this question is to contrast how probe distractors in each task are held to function.

Taking this approach, the current results here are consistent with the idea that the probe-trial distractor in location-based tasks has a motivational and a retrieval role, revealed by the 'SNP remove' - 'SNP restore' pattern seen here and reported by Fitzgeorge and Buckolz (2008). The certain or highly likely absence of a probe distractor can motivate the disengagement of the SNP process, evident on target-only probe trials (SNP removal), presumably by blocking the retrieval of the stored representations of prime-trial distractor processing. This blocking can be circumvented; however, and so the SNP effect is restored by presence of a probe distractor event that independently triggers the retrieval of stored distractor representations, providing its identity fully matches the identity of the prime distractor. Importantly, the presence of a probe distractor does not influence retrieval because it causes a matching of event numbers on the prime and probe trials; SNP can be present when event numbers mismatch (Buckolz et al., 2008; Neill et al., 1994), and can be absent when they match (Table 5). In short, retrieval is identity and not event-number based in location tasks.

Looking at identity NP, event number matching on consecutive prime and probe trials (Moore, 1994) was, again, not a perquisite for distractor representation retrieval; event number matching did not guarantee an identity NP effect (Moore) and so the probe distractor, as in the SNP task, was not influential in identity NP production on this account. 
In contrast, the critical feature of a probe distractor in identity tasks on NP appearance is that it causes (or appears to cause) a response conflict (Moore, 1994); such an impact is unimportant in SNP tasks (Table 5). This distinction would explain why the actual absence of a probe distractor in identity NP tasks (i.e., no conflict), irrespective of the likelihood of this absence, is typically associated with the lack of an NP effect (Moore), while this is not so with SNP tasks. SNP effects can still be observed when the probe is distractor free (Neill et al., 1994).

Finally, the motivational and direct retrieval roles of the probe distractor in SNP tasks are not evident for probe distractors in identity NP tasks. Rather, the retrieval role of the probe distractor in the latter task is indirect, somehow occurring because a response conflict causes the maintenance of an inhibitory state (Moore, 1994). This is consistent with the fact that the prime and probe distractors in identity NP tasks often totally mismatch, and yet identity NP is produced. Oddly enough, identity matching of prime and probe trial distractor events is unimportant to retrieval in an identity task, but is instrumental in a location task.

If the processing roles of the probe distracters in the location and identity NP tasks noted here are correct, the underlying processing for these NP phenomena differ in this sense. Incidentally, the fact that identity (e.g., Connelly \& Hasher, 1993, Ex. 3), but not location, NP effects are lost as we age (Lok, 2012). This is consistent with the above in pointing to some different underlying processing for these two phenomena. In the case of aging, however, the preservation of inhibitory after-effects (e.g., SNP) in location-based tasks might be related to the fact that they are caused by response inhibition (Guy et al., 2006), which acts as a late filter to prevent action chaos. Given the importance of this role, it is reasonable that response inhibition, and so its related inhibitory after-effects, do not materially decline with age. This possibility is supported by Maylor, Birak, and Schlaghecken (2011) who showed that an inhibitory after-effect produced by response inhibition in an identity task (i.e., the negative 
compatibility effect) was preserved in older adults (note- their prime distracters were masked). Accordingly, we should see no age-related decrement in identity NP when the task exhibits are response inhibition locus.

\section{Storage of Prime-trial Processing Representations}

To our knowledge, we saw here the first evidence that representations of prime trial processing in location-based tasks are stored episodically; the removal of the SNP effect coincided with a removal of target-repeat reaction time reductions. Neither prime target nor prime distractor processing representations were retrieved. We should see in future work if the lack of a target-repeat effect caused by target identity mismatches between the prime and probe trials is accompanied by the removal of an SNP effect. In any event, Fox and De Fockert (1998) did show that prime trial representations in identity NP tasks were also stored episodically; changing the background colour of either the prime or the probe trials removed identity NP. Despite this storage format similarity, as we noted earlier, the contents of the stored episodes for location and identity tasks seem to qualitatively differ. Distractor event identity, important for representation retrieval in location tasks, is not a factor in identity tasks.

Finally, in a different vein, bear in mind that the SNP data obtained and referenced here relate to those procedures using visual events that were centrally presented. The latter is important because such central event presentations presumably avoid contamination of response inhibition after-effects from those produced by orientation inhibition effects (e.g., Buckolz et al., 2012a), held to cause the inhibition of return phenomenon (e.g., Klein, 2000). Furthermore, the visual distinction is appropriate to bear in mind because the use of different sensory systems may yield different distractor processing and related inhibitory after-effects causes; as Moller, Mayr, and Mueller (2013) have demonstrated for auditory and visual SNP. 


\section{Limitations of the Current Study and Future Research}

In the present study, we used a 'remove SNP' - 'restore SNP' approach (Fitzgeorge \& Buckolz, 2008) as a means of determining whether the presence of a probe trial distractor event results in the retrieval of stored representations of earlier (prime trial) distractor processing. We used the infrequent occurrence of a probe distractor event (i.e., .25 [probe distractor present]/.75 [probe distractor absent]) to motivate individuals to disengage the SNP process (i.e., remove SNP). Once successfully achieved, we could test the impact of primeprobe distractor identity similarity levels on distractor representation retrieval, evidenced by the 'restoration' of an SNP effect. As it turned out, the success rate of the $.25 / .75$ manipulation in producing SNP disengagement among our subjects was at about the 50\% level; this in spite of our procedural efforts to have the disengagement effectiveness of the $.25 / .75$ procedure to be 'high'. Concern about the low success rate of the $.25 / .75$ procedure here rested with the fact that it did reduce the number of subjects we had available for testing the 'restore SNP' behaviour; otherwise, it was not disconcerting, although it did focus on the need to find better ways to induce SNP disengagement over subjects.

Toward this end, future work, aimed at ultimately looking into the retrieval role of probe distractor events (i.e., 'restore SNP'), could first look to see if increasing the probability imbalance of 'probe distractor present' vs. 'probe distractor absent' trials to see if this would increase the percentage of subjects showing SNP disengagement (e.g., .20/.80 or even higher, respectively, being mindful of the increase in the number of trials that would needed to run a study as the imbalance increased).

In addition, one could induce SNP disengagement in another way, by cueing the impending likelihood of an ignored-repetition trial (see Fitzgeorge \& Buckolz, 2008), with the hope that it would motivate SNP disengagement in a larger number of recruited subjects. It is important to note this future work is directed at understanding how SNP 
removal/disengagement can be more universally induced in sampled individuals. It does not, by itself, deal with the question of whether probe trial distracters have a retrieval role.

Some additional future experimentation was suggested by some of our unexpected findings. In particular, the preliminary indication here is that prime trial distractor and target events in a location-based task may be stored episodically, that is as a unit (Neill, 2007). If so, when retrieval of the stored representations about one of these events is indicated in the data, we should also see evidence of retrieval of the other events stored representations. In the experiment reported here, this pattern was evident. Conditions that showed that prime distractor representations had not been retrieved also showed that this retrieval failure applied to prime target representations as well. For example, when the prime trial and the probe trial distractor identities mismatched; neither an SNP effect, nor a beneficial effect associated with target-repeat trials, were evident, indicative of the lack of retrieval of for either of the prime distractor or prime target events. In our study, prime-probe target identities matched while prime-probe distractor identities could match or mismatch. A further testing of the episodic storage of prime distractor and target events would see the reverse occurring, whereby primeprobe distractor identities would match while the prime-probe target event identities could match or mismatch. Support for the episodic storage of prime trial event processing would see the presence of after-effects (i.e., SNP effect, target-repeat RT facilitation) when the prime-probe trial target identities matched, but not when they mismatched. Such a result would also indicate that the retrieval role played by a probe distractor may simply be to complete the 'episode' on the probe trial; that is, by having both the target and the distractor prime-probe identities match. This would trigger the retrieval of stored prime trial processing. Essentially, then, the probe distractor would not have its own 'retrieval route' as proposed by Buckolz et al. (2012). Rather its impact on retrieval (i.e., restore SNP) comes from fulfilling the retrieval requirements of episodically stored events, full matching of event identities 
between prime and probe trials. As noted earlier, if this is so, having the target identities mismatch from prime to probe trials should prevent retrieval (event when prime-probe distractor identities match), since a full matching of prime to probe events would be obviated. 


\section{References}

Bauer E., Gebhardt H., Ruprecht C., Gallhofer B., Sammer G. (2012). Functional MRI evidence for episodic retrieval and inhibition in negative priming. PLoS ONE 7:e36089. doi: 10.1371/journal.pone.0036089.

Buckolz, E., Avramidis, C., \& Fitzgeorge, L. (2008). Prime-trial processing demands and their impact on distractor processing in a spatial negative priming task. Psychological Research, 72, 235-248.

Buckolz, E., Boulougouris, A., \& Khan, M. (2002). The influence of probe-trial selection requirements on the location negative priming effect. Canadian Journal of Experimental Psychology, 56, 274-283.

Buckolz, E., Edgar, C., Kajaste, B., Lok, M., \& Khan, M. A. (2012) Inhibited prime-trial distractor responses solely produce the visual spatial negative priming effect. Attention, perception \& psychophysics, 74(8), 1632-1643.

Buckolz, E., Fitzgeorge, L., \& Knowles, S. (2011). Spatial negative priming, but not inhibition of return, with central (foveal) displays. Psychology, 3, 666-674.

Buckolz, E., Goldfarb, A., \& Khan, M. (2004). The use of a distractor-assigned response slows later responding in a location negative priming task. Perception \& Psychophysics, 66, 837-845.

Chao, H.-F. (2009). Revisiting the role of probe distractors in negative priming: Location negative priming is observed when probe distractors are consistently absent. Attention, Perception, \& Psychophysics, 71, 1072-1082. 
Chao, H.-F. (2011). Dissociations between identity and location negative priming. Acta Psychologica, 136(1), 81-89.

Christie, J. \& Klein, R. M. (2001) Negative Priming for spatial location? Canadian Journal of Experimental Psychology. 55, 24-38.

Connelly, S. L., \& Hasher, L. (1993). Aging and the inhibition of spatial location. Journal of Experimental Psychology: Human Perception and Performance, 19, 1238-1250.

Fitzgeorge, L. \& Buckolz, E. (2009). Automatic versus volitional orienting and the production of the inhibition-of-return effect. Canadian Journal of Experimental Psychology, 63, 94-102.

Fitzgeorge, L., \& Buckolz, E. (2008). Spatial negative priming modulation: The influence of probe-trial target cueing, distractor presence and an intervening response. European Journal of Cognitive Psychology, 20, 994-1026.

Fitzgeorge, L., Buckolz, E., \& Khan, M. (2011). Recently inhibited response are avoided for both masked and nonmasked primes in a spatial negative priming task. Perception and Psychophysics, DOI 10.3758/s13414-011-0125-7.

Fox, E., \& DeFockert, J. (1998). Negative priming depends on prime-probe similarity: Evidence for episodic retrieval. Psychonomic Bulletin, 5, 107-113.

Graboi, D. G. (1971) Searching for targets: The effects of specific practice. Perception and Psychophysics 10, 300-304.

Guy, S., \& Buckolz, E. (2007). The locus and modulation of the location negative priming effect. Psychological Research, 71, 178-191. 
Guy, S., Buckolz, E., \& Fitzgeorge, L. (2007). Disengaging the location negative priming effect: The influence of an intervening response. European Journal of Cognitive Psychology, 19, 789-812.

Guy, S., Buckolz, E., \& Khan, M. (2006). The locus of location repetition latency effects. Canadian Journal of Experimental Psychology, 60, 307-318.

Guy, S., Buckolz, E., \& Pratt, J. (2004). The influence of distractor-only prime trials on the location negative priming mechanism. Experimental Psychology, 51, 4-14.

Houghton, G. \& Mari-Beffa, P. (2005). Dissociations between object- and response-based components of negative priming. Perception and Psychophysics. 67(8), 1423-143.

Houghton, G., \& Tipper, S. P. (1994). A model of inhibitory mechanisms in selective attention. In D. Dagenbach \& T. H. Carr (Eds.), Inhibitory processes in attention, memory, and language (pp. 53-112). San Diego: Academic Press.

Klein, R. M. (2000). Inhibition of return. Trends in Cognitive Sciences, 4, 138-147.

Klein, R., Christie, J., \& Morris, E. P. (2005). Vector averaging of inhibition of return. Psychonomic Bulletin \& Review, 12(2), 295-300.

Lok, M. (2011). On the preservation of response inhibition and free choice behaviour in the elderly for location-based tasks. Unpublished MA thesis, University of Western Ontario, London, Ontario, Canada.

Maylor, E. A., Birak, K. S., \& Schlaghecken, F. (2011). Inhibitory motor control in old age: evidence for de-automatization? Frontiers in Psychology, 2, 1-9. 
Milliken, B., Tipper, S. P., Houghton, G., \& Lupianez, J. (2000). Attending, ignoring, and repetition: On the relation between negative priming and inhibition of return. Perception \& Psychophysics, 62, 1289-1296.

Milliken, B., Tipper, S.P., \& Weaver, B. (1994). Negative priming in a spatial localization task: Feature mismatching and distractor inhibition. Journal of Experimental Psychology: Human Perception and Performance, 20, 624-646.

Moller, M., Mayr, S., \& Buchner, A. (2013). Target localization among concurrent sound sources: no evidence for the inhibition of previous distractor responses. Attention, Perception \& Psychophysics, 75(1), 132-44.

Moore, C. (1994). Negative priming depends on probe-trial conflict. Perception and Psychophysics, 56(2), 133-147.

Neill, W. T. (1997). Episodic retrieval in negative priming and repetition priming. Journal of Experimental Psychology: Learning, Memory, and Cognition, 23, 1291-1305.

Neill, W. T. (2007). Mechanisms of transfer-inappropriate processing. In D. S. Gorfein \& C. M. MacLeod (Eds.), Inhibition in Cognition (pp. 63-78). Washington D. C.: American Psychological Association.

Neill, W. T., Terry, K. M., \& Valdes, L. A. (1994). Negative priming without probe selection. Psychonomic Bulletin and Review, 1, 119-121.

Neill, W. T., Valdes, L. A., Terry, K. M., \& Gorefin, D. S. (1992). Persistence of negative priming: II. Evidence for episodic trace retrieval. Journal of Experimental Psychology: Learning, Memory, \& Cognition, 18, 993-1000. 
Park, J., \& Kanwisher, N. (1994). Negative priming for spatial locations: Identity mismatching, not distractor inhibition. Journal of Experimental Psychology: Human Perception and Performance, 20, 613-623.

Rafal, R., Davies, J., \& Lauder, J. (2006). Inhibitory tagging at subsequently fixated locations: Generation of "inhibition of return" without saccade inhibition. Visual Cognition, 13, 308-323.

Stoddart, A. (2013). The error protection impact of inhibitory after-effects in location-based tasks and their preservation with practice. Unpublished MA thesis, University of Western Ontario, London, Ontario, Canada.

Sumner, P. (2007). Negative and positive masked-priming - implications for motor inhibition. Advances in Cognitive Psychology, 3, 317-326.

Tipper, S. P. (2001). Does negative priming reflect inhibitory mechanisms? A review and integration of conflicting views. The Quarterly Journal of Experimental Psychology, $54 A, 321-343$.

Tipper, S. P., Weaver, B., Cameron, S., Brehaut, J. C., \& Bastedo, J. (1991). Inhibitory mechanisms of attention in identification and localization tasks: Time course and disruption. Journal of Experimental Psychology. Learning Memory And Cognition, 17(4), 681-692.

Valle-Inclan, F., \& Redondo, M. (1998). On the automaticity of ipsilateral response activation in the Simon effect. Psychophysiology, 35, 366-371. 
Ignored Repetition (IR): A probe trial target stimulus that arises at a location previously occupied by the distractor event on the prime trial.

Control (CO): A probe target stimulus that appears at a location previously unused by the prime trial events.

Target Repetition (TR): A probe trial target stimulus that appears at a location previously occupied by the target event on the prime trial.

Execution Resistance (ER): A property of distractor response processing that repels the execution of a just inhibited response.

Inhibitory After-effects (IAE): Execution resistance exerts an influence on future processing causing such effects.

Spatial Negative Priming (SNP): Slower reaction times when responding to a target stimulus that arises at a location previously occupied by a distractor event (ignored-repetition [IR] trial) than when it appears at a recently unused location (control [CO] trial). 
Appendix A

Pilot study 


\section{Pilot study}

Purpose

The details of the pilot study will be highlighted only briefly, restricted to those aspects that have theoretical or procedural implications for the main study reported here. The purpose of the pilot study was to again test the ability of a .25 (probe distractor present)/.75 (probe distractor absent) probability imbalance to motivate the disengagement of the spatial negative priming (SNP), and to then examine the SNP restoration impact of probe trial distractor events. In the latter regard, we examined the impact of prime-probe distractor identity matching levels (full match, partial match, total mismatch) on SNP restoration.

Perhaps of some procedural importance, the distractor identity matching levels were intermixed within a trial series in the pilot study. (See Table 6)

\section{Method}

\section{Participants}

Thirty university undergraduate students (19 males, 11 females), from the School of Health Sciences, Western University, participated in the study. Participant's age ranged from $20-24$ years and with normal or corrected-to-normal vision, participated in this experiment. Participants were randomly chosen and requested to read and sign the ethics consent form prior to participation.

\section{Apparatus}

The apparatus was the same as in the main experiment 


\section{Procedure}

All procedures were the same as in the main experiment with the exception of those noted below. The delay of the stimulus onset asynchrony (SOA) was $500 \mathrm{~ms}$, which is the delay of the prime response to the presentation of the probe stimulus (panels 4 to 5 on Schematic 3). The shortening of the SOA has no direct implication on the current results as the shorter interval does not affect the production of the spatial negative priming effect (Buckolz, Boulougouris, Khan, 2002).

Three factors were manipulated: Group (prime-probe distractor matching level of the partial match; either colour [Group 1] or shape [Group 2]. Probe distractor conditions (primeprobe distractors fully matched, partially matched, totally mismatch or distractor absent), and trial-type (IR: ignored repetition, CO: control). The first of these was a between-subjects factor. Participants completed two conditions which consisted of four experimental sessions of approximately 30 minutes each. Participants were randomly assigned into two groups and began each condition in a counter-balanced manner. Details of each condition and primeprobe distractor matching levels can be found in Table 6 .

When a target and/or distractor appeared on the prime and probe trials, they did so randomly at all possible locations. This resulted in 1728 trial pairs: 396 IR trials (one location re-used, probe target-to-prime distractor location), 396 TR trials (one location re-used, probe target-to-prime target location) and $720 \mathrm{CO}$ trials (no locations were re-used). The remaining prime-probe event combinations occurred on 216 trials (not used for analysis). 
Distractor identity matching levels (full match, partial match, total mismatch: within-subjects), each produced in two different conditions by using different prime and probe trial distractor identities (within-subjects).

Condition 1

Probe

Distractor Condition: Prime

Red rectangle

Red rectangle

Red rectangle

Red rectangle

Group 2

Partial Match:

Group 1

Group 2

Red rectangle

Blue rectangle

Red rectangle

Red triangle

Total Mismatch:

Group 1

Group 2

Red rectangle

Yellow cross

Red rectangle

Yellow cross

Absent:

Group 1

Group 2

Red rectangle

Red rectangle

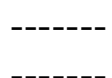

Distractor Condition:

Prime

\section{Probe}

Full Match:

Group 1

Group 2

Blue rectangle

Red triangle

Blue rectangle

Partial Match:

Group 1

Group 2

Blue rectangle

Red triangle

Red rectangle

Blue rectangle

Total Mismatch:

Group 1

Group 2

Blue rectangle Yellow cross

Red triangle Yellow cross

Absent:

Group 1

Group 2
Blue rectangle

Red triangle

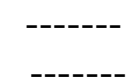

30 participants completed both conditions as assigned to their respective groups. All subjects experienced three types of prime-probe distractor identity matching levels within one condition. 


\section{Results}

We calculated an ANOVA using mean subject reaction times (RT) with Distractor Identity Similarity level (full match, partial match, total mismatch, absent) and Probe Trial Type (ignored-repetition [IR], control [CO]) as the main factors (see Table 6). Separate ANOVAs were performed for each Group and Condition and mean RTs can be found in Table 7.

Condition 1. Group 1 Probe Trial Type factor yielded a significant SNP effect (15 $\mathrm{ms}), F(1,14)=14.92, \underline{p}<0.01, M S E=447$; however, more importantly, this factor did not interact with Distractor Identity Similarity Levels, $\underline{p}=0.13$. Therefore, the SNP effect was present on distractor-free probe trials (15 ms). Similarly, Group 2 analysis produced a Probe Trial Type significant SNP effect $(17 \mathrm{~ms}), F(1,14)=76.81, \underline{p}<0.01, M S E=121$; notably, this did not interact with Distractor Identity Similarity Levels, $\underline{p}=0.82$. Therefore, the SNP effect was present on distractor-free probe trials $(14 \mathrm{~ms})$. These finding are unlike previous work on disengagement where a .25 (distractor present)/ .75 (distractor absent) probe distractor manipulation produced modulation affects (Fitzgeorge \& Buckolz, 2008).

Condition 2. Group 1 Probe Trial Type factor was non-significant $(8 \mathrm{~ms}), F(1,14)=$ 3.00, $\underline{p}=0.11, M S E=611$. Additionally, this factor did not interact with Distractor Identity Similarity Levels, $\underline{p}=0.88$. Therefore, it would seem that probe distractor manipulation of $.25 / .75$ motivated disengagement of the SNP process. However, SNP processes are not restored with the presence of a probe distractor giving no meaningful explanation to the restorative impact of probe Distractor Similarity Levels. Group 2 Probe Trial Type factor yielded a significant SNP effect $(11 \mathrm{~ms}), F(1,14)=13.95, \underline{p}<0.01, M S E=280$; however, more notably, this factor did not interact with Distractor Identity Similarity Levels, $\underline{p}=0.74$. Therefore, the SNP effect was present on distractor-free probe trials (10 ms). 
Planned comparisons of the Probe Trial Type (IR vs. CO) on distractor absent probe trials were conducted as it was hypothesized that a $.25 / .75$ manipulation would disengage the SNP process on distractor-free probe trials (Fitzgeorge \& Buckolz, 2008). A t-test investigating the RT differences between IR and CO trials (i.e., SNP = $15 \mathrm{~ms}$ ) for distractorfree probe trials elicit a statistically significant effect, $t(14)=6.12, \underline{p}<0.01, S E=2.44$ for Condition 1: Group 1. This was also similar in both Conditions for Group 2, $t(14)=7.02, \underline{p}$ $<0.01, S E=2.00(\mathrm{SNP}=14 \mathrm{~ms})$, and $t(14)=4.28, \underline{p}<0.01, S E=2.34(\mathrm{SNP}=10 \mathrm{~ms})$ respectively. Accordingly, the $.25 / .75$ manipulation was unsuccessful in motivating disengagement. Thus, it was not possible to examine the main issue; namely, do probe trial distractor restore an otherwise absent SNP effect, and, if so, is SNP restoration dependent upon the identity similarity level of the prime and probe distracters.?

\section{Implications}

The purpose of the pilot study was to motivate the disengagement of the SNP process by using a .25 (distractor present)/.75 (distractor absent) probability manipulation, so we could examine the 'SNP restoration' power of the probe distractor. Overall, the $.25 / .75$ probe distractor manipulation did not achieve its goal of SNP disengagement in the pilot study. One possible reason for this lack of success was that the pilot study had the distractor identity matching levels vary from trial to trial within a trial sequence. This contrasted with Fitzgeorge and Buckolz (2008), who had overall success with the $.25 / .75$ probe distractor manipulation, and whose distractor identity matching level was fixed (full match), and so known it advance.

It is possible that the intermixing of the distractor identity matching levels within a session interferes with the subjects' overall perception of, or belief in, the probe distractor probability imbalance in place. In turn, this could interfere with a subject's willingness to 
disengage the SNP process. We tested this possibility in the main experiment where the prime-probe distractor identity matching levels (full match, partial match, total match, etc.) were fixed within a session. 
Table 7

Mean reaction times $(R T, \mathrm{~ms})$ for the Trial Type (ignored-repetition, control, target-repeat) and Distractor Identity Matching levels (full match, partial match, total mismatch) factors

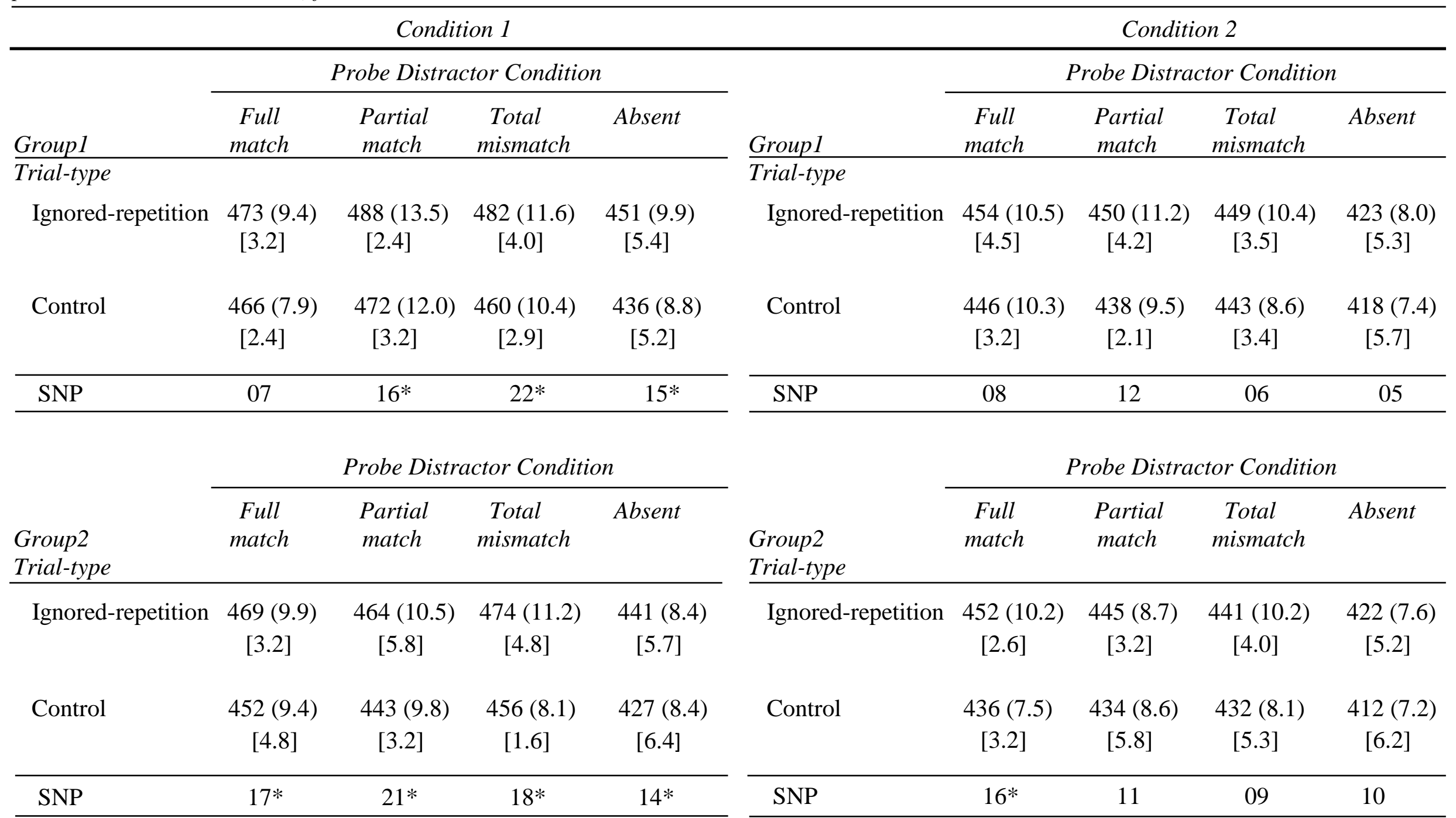

Note. Spatial Negative Priming = Ignored-repetition - Control. $(\mathrm{f})=$ standard error $(\mathrm{ms}) ;\left[=\right.$ button press error percentage. ${ }^{*} p<0.05 . n=30$ per group between conditions. 
Appendix B

The University of Western Ontario Research Ethics Board of Approval Notice 


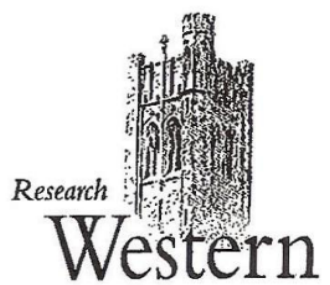

Use of Human Participants - Ethics Approval Notice

Principal Investigator: Dr. Eric Buckolz

Review Number: 15180s

Review Level: Delegated

Approved Local Adult Participants: 300

Approved Local Minor Participants: 0

Protocol Tisle: Properties of Inhibitory After-effects

Department \& Institution: Kinesiology, University of Westem Ontario

Sponsor: Natural Sciences and Engineering Research Council

Ethics Approval Date: July 13, 2011

Expiry Date: July 31, 2013

Documents Reviewed \& Approved \& Documents Received for Information:

Document Name Comments

Version

Date

Revised Study End The study end date has been revised to July 30, 2013 to allow

Date for project completion.

This is to notify you that The University of Western Ontario Research Ethics Board for Non-Medical Research Involving Human Subjects (NMREB) which is organized and operates according to the Tri-Council Policy Statement: Ethical Conduct of Research Involving Humans and the applicable laws and regulations of Ontario has granted approval to the above referenced revision(s) or amendment(s) on the approval date noted above.

This approval shall remain valid until the expiry date noted above assuming timely and acceptable responses to the NMREB's periodic requests for surveillance and monitoring information. If you require an updated approval notice prior to that time you must request it using the UWO Updated Approval Request Form.

Members of the NMREB who are named as investigators in research studies, or declare a conflict of interest, do not participate in discussions related to, nor vote on, such studies when they are presented to the NMREB.

The Chair of the NMREB is Dr. Riley Hinson. The UWO NMREB is registered with the U.S. Department of Health \& Human Services under the IRB registration number IRB 00000941.

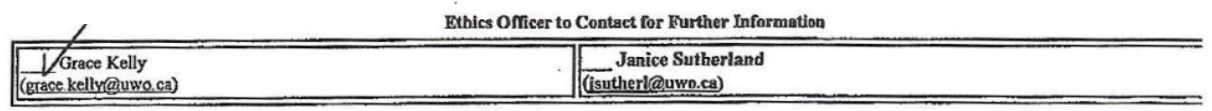

This is an official document Please retain the original in your files

The University of Western Ontario

Office of Research Ethics

Support Services Building Room 5150 - London, Ontario • CANADA - N6A 3K7

PH: 519-661-3036•F: 519-850-2466•ethics@uwo.ca • www.uwo.ca/research/ethics 
Appendix $C$

Letter of Information \& Script 


\section{Letter of information \& Script}

\section{Letter of Information}

Project Title: Properties of Inhibitory After-effects

\section{Introduction}

You are being invited to participate in a research study. The purpose of this letter is to provide you with the information you need to render an informed participation decision.

\section{Purpose of the Study}

The purpose of the study is to extend our understanding of one as aspect of cognitive 'inhibitory after-effects', which refer to those occasions where a current act of inhibition results in interference effects (i.e., delayed responding time, error production) upon future processing in which the inhibited events participate. Inhibition is synonymous with the term 'prevention.' It refers to preventing the processing of various stimuli or the execution of various responses that we do not wish to do.

\section{Basic Procedures}

If you agree to participate, you will be asked to react as quickly as possible to visual target stimuli presented on a computer screen while concurrently ignoring distractor events that may also be present. You will respond to the spatial location and/or to the identity of stipulated target stimuli by pressing designated computer keyboard buttons. Both the accuracy (button press errors) and decision times (reaction times) associated with your manual button press responses will be recorded and analyzed.

The general purpose of this experimentation is to extend our understanding of cognitive inhibition, which relates to our ability to prevent the unwanted processing of visual (distractor) information and/or their associated responses.

Participation requires you to attend multiple testing sessions in laboratories located in <blank>. Specific laboratory testing times will be arranged by you in consultation with the Experimenter who can be contacted by email or by phone

\section{Risks Associated with Participation}

There are no known or reasonably anticipated risks associated with participation. 


\section{Benefits}

No personal benefits will necessarily follow from your participation. It is possible, however, that your experience with, and understanding of, reaction time type tasks, along with knowledge gained from a debriefing session where the results obtained and their implications are noted will be viewed by you as beneficial. Additionally, any discoveries that advance our understanding of 'inhibitory after-effects' as a result of your participation might be viewed by yourself as a benefit.

\section{Confidentiality}

Efforts will be made to ensure that your data cannot be linked to you personally by anyone other than the Experimenter. Code numbers assigned to your data files will not identify you directly but will be linked to your name on a master sheet kept by the Experimenter on a password protected computer. Once experimentation has been completed, the master sheet will be destroyed. Henceforth, it will be impossible to associate any particular data with your identity.

The data files and the master sheet will be stored on separate, password-protected computers located in locked laboratory or office spaces that are accessible only to the Experimenter. Publications that might arise from the data collected will not identify you personally. The data files will be retained for 5 years in the event publication does not arise, or for 5 years after 'online' publication, and then deleted.

\section{Participation}

Participation in this study is voluntary. You may refuse to participate or withdraw from the study at any time without penalty. If you withdraw, any data collected to that point will be deleted and will not be used in the study.

\section{Debriefing}

Once all of the data collection has been completed, you may contact the Experimenter by email for an explanation of the purpose of the study, along with the preliminary findings obtained. A debriefing session will also take place in the laboratory once all of the data have been collected which you can attend. At that time, information dealing with your participation will be discussed (i.e., study purpose, group results and their preliminary interpretation). The timing of the debriefing session will be told to you after your last testing session, or you can later email the Experimenter for this information. 


\section{Contact Information}

You do not waive any legal rights by signing the Consent Form.

This letter is yours to keep.

\section{Script}

On the screen four lines will appear separated by a cross in the middle. The fixation cross is what you constantly want to focus on. Rectangles will flash above these lines. You want to respond to the green ones (targets), while ignoring the red rectangles OR blue rectangles OR yellow cross for example (distractors). You can respond by pressing the letter that corresponds to its assigned location. So line 1 corresponds with "D", line 2 corresponds with "V", line 3 corresponds with "M" and line 4 corresponds with "L".

Trials will appear in pairs, first the prime and then the probe. At the beginning of each pair you will hear a tone "beep" followed by the appearance for the four lines and cross. Once the cross appears this is where you should focus your attention. Always focus on the cross. Anytime a green rectangle appears, you want to respond with the correct button, again anytime a distractor (any colour OR shape) appears you want to ignore it. Once you respond with the correct response, the rectangles will disappear and the next trial will start. There will be times when both target and distractor appear, simply ignore the distractor \& respond to the green. However, on the second presentation (probe trial) the probability of the distractor appearing with a target is low $(.25 \%)$ where a target-only trial will appear for the remainder of the trials $(.75 \%)$.

After several trials something will pop up saying you deserve a break, to continue just press the space bar and the trials will resume. At the end, there will be a pop up saying "Congratulations you're done." You don't have to do anything, just leave the computer and come and get me.

Two important things to remember: You want to respond as quickly as possible while minimizing errors, and you want to make sure that you don't anticipate (don't respond before the stimuli appear).

Any questions? We'll do a few practice trials so you can get the hang of it (10 practice trials or until student has full understanding). 


\section{Curriculum Vitae \\ SECTION I \\ Personal Information}

NAME:

ADDRESS:

WORK ADDRESS:

CONTACT:

\section{SECTION II} Education

\section{POST-SCEONDARY \\ EDUCATION AND \\ DEGREES:}

HONOURS AND AWARDS:
The University of Western Ontario London, Ontario, Canada Masters of Arts, Kinesiology Supervisor: Dr. Eric Buckolz 2013

The University of Wales, Bangor Bangor, Wales, United Kingdom

Bachelor of Science, Honours Sport, Health and Physical Education 2011

Bury College

Bury, Lancashire, England, United Kingdom

A-Level, Triple Distinction BTEC National Diploma in Sport 2008

The Faculty of Health Sciences: University of Western Ontario Value: $\$ 16700$

2012 - 2013

Outstanding Thesis in Motor Leaning: University of Wales, Bangor Value: $£ 500$

2011 
Sport Scholarship: University of Wales, Bangor

Value: $£ 500$

$2008-2011$

Bury College Scholar

Value: $£ 100$

2008

\title{
Section III
}

Academic Presentations \& Societies

\section{CONFERENCE PRESENTATION AND POSTERS}

2012

Buckolz, E., Stoddart, A., Kajaste, B., Edgar, C., \& Haworth, P. (2012, November). The Influence of Practice on Response Inhibition (Distractor Potency) and Its Inhibitory After-effects in Visual, Location-based Tasks. Poster presented at the Canadian Society for Psychomotor Learning and Sport Psychology Conference, Halifax, Nova Scotia.

Stoddart, A., Buckolz, E., Edgar, C., Kajaste, B., \& Haworth, P. (2012, November). Protection Against Response Selection Errors: Is this the Benefit Produced by Inhibitory After-effects Caused by Response Inhibition?. Poster presented at the Canadian Society for Psychomotor Learning and Sport Psychology Conference, Halifax, Nova Scotia

\section{MEMBERSHIP IN ACADEMIC OR PROFESSIONAL SOCIETIES}

2012 - Present Canadian Society for Psychomotor Learning and Sport Psychology Conference

2012 - Present $\quad$ Society of Graduate Students (SOGS)

\section{Section IV \\ Work Experience \& Professional Qualifications}

January 2012 - Present

\author{
University of Western Ontario \\ Teaching Assistant \\ Module: Psychomotor Behaviour - Dr. Mathew Heath \\ Examination supervision and marking \\ Helping and advising undergraduate students \\ Soccer Official \\ Referring intramurals \\ Ensuring a save playing environment
}


November 2009 - 2011

\section{Menai Track and Field Athletics Club}

Junior Endurance Athletics coach (voluntary work)

Provided athletic sessions to 8-15 years olds

Coached and inspired young people in athletics by an increase number of participation in my sessions.

Improved coaching/teaching skills whilst working independently

November $2005-2011$

\section{ASDA Wal-Mart, Pilsworth, Llangefni}

Sales Assistant, Training Coordinator

Negotiated with difficult customers

Supervised new colleagues

Planned and coordinated new shop floor arrangements

Allocated jobs to other fellow colleagues

Summer 2007 - 2009

\section{Siddal Moor Sports College}

Teacher assistant (voluntary work)

Produced lesson plans to improve organisation and written

communication skills

Provided sessions to 11-16 year olds

Improved teaching skills by shadowing other teachers

Established a good teacher/pupil relationship

\section{PROFESSIONAL QUALIFICATIONS}

COACHING:

United Kingdom Athletics (UKA) Level 1 \& 2

Completed: 2010

Lawn Tennis Association (LTA) UKCC Level 1 \& 2

Completed: 2010

Basketball UKCC Level 1

Completed: 2010

ASA Swimming Level 1

Completed: 2010 


\section{Section V \\ Publications}

Haworth, P., Buckolz, E., \& Kajaste, B. (under review). The Role of Probe-trial Distractors in the

Production/Removal of the Spatial Negative Priming Effect. Journal of Cognitive Psychology 